\title{
LABOCA mapping of the infrared dark cloud MSXDC G304.74+01.32^
}

\author{
O. Miettinen and J. Harju
}

\author{
Department of Physics, PO Box 64, 00014 University of Helsinki, Finland \\ e-mail: oskari.miettinen@helsinki.fi
}

Received 13 November 2009 / Accepted 18 March 2010

\section{ABSTRACT}

\begin{abstract}
Context. Infrared dark clouds (IRDCs) likely represent very early stages of high-mass star/star cluster formation.
Aims. The aim is to determine the physical properties and spatial distribution of dense clumps in the IRDC MSXDC G304.74+01.32 (G304.74), and bring these characteristics into relation to theories concerning the origin of IRDCs and their fragmentation into clumps and star-forming cores.

Methods. G304.74 was mapped in the $870 \mu \mathrm{m}$ dust continuum with the LABOCA bolometer on APEX. The $870 \mu \mathrm{m}$ map was compared with the $1.2 \mathrm{~mm}$ continuum map of the cloud by Béltran et al. (2006, A\&A, 447, 221). Archival MSX and IRAS infrared data were used to study the nature and properties of the submillimetre clumps within the cloud. $\mathrm{The}_{2} \mathrm{H}_{2}$ column densities were estimated using both the $870 \mu \mathrm{m}$ dust emission and the MSX $8 \mu \mathrm{m}$ extinction data. The obtained values were compared with near-infrared extinction which could be estimated along a few lines of sight. We compared the clump masses and their spatial distribution in G304.74 with those in several other recently studied IRDCs.

Results. Twelve clumps were identified from the $870 \mu \mathrm{m}$ dust continuum map. Three of them are associated with the MSX and IRAS point sources. Moreover, one of the clumps (SMM 6) is associated with two MSX $8 \mu \mathrm{m}$ point-like sources. Thus, there are 8 clumps within G304.74 which are not associated with mid-infrared (MIR) emission. The $\mathrm{H}_{2}$ column densities derived from the dust continuum and extinction data are similar. The comparison suggests that the dust temperature may be elevated (20-30 K) near the southern end of the cloud, whereas the starless clumps in the centre and in the north are cool $\left(T_{\mathrm{d}} \sim 15 \mathrm{~K}\right)$. There is a high likelihood that the clump mass distributions in G304.74 and in several other IRDCs represent the samples of the same parent distribution. In most cases the spatial distributions of clumps in IRDCs do not deviate significantly from random distributions.

Conclusions. G304.74 contains several massive clumps that are not associated with MIR emission. On statistical grounds it is likely that some of them are or harbour high-mass starless cores (HMSCs). The fact that the clump mass distributions (resembling the highmass stellar IMF), and in some cases also the random-like spatial distributions, seem to be comparable between different IRDCs, is consistent with the idea that the origin of IRDCs, and their further sub-fragmentation down to scales of clumps is caused by supersonic turbulence in accordance with results from giant molecular clouds.
\end{abstract}

Key words. stars: formation - ISM: clouds - ISM: structure - radio continuum: ISM - submillimeter: ISM

\section{Introduction}

\subsection{Infrared dark clouds}

The so-called infrared dark clouds (IRDCs) were discovered by the Infrared Space Observatory (ISO; Pérault et al. 1996) and the Midcourse Space Experiment (MSX; Egan et al. 1998); the clouds were detected as dark absorption objects against the bright mid-infrared (MIR) Galactic background radiation. Based on the MSX $8 \mu \mathrm{m}$ data of the Galactic plane from $l=0-360^{\circ}$ and $|b| \leq 5^{\circ}$, Simon et al. (2006a) identified almost 11000 IRDC candidates. Recently, Peretto \& Fuller (2009) used Spitzer satellite data to extract about 9000 new IRDC candidates.

Molecular line and dust continuum studies of IRDCs have shown that they are cold $(T<25 \mathrm{~K})$, dense $\left(n\left(\mathrm{H}_{2}\right) \gtrsim 10^{5} \mathrm{~cm}^{-3}\right.$, $\left.N\left(\mathrm{H}_{2}\right) \gtrsim 10^{22} \mathrm{~cm}^{-2}\right)$, and massive $\left(\sim 10^{2}-10^{5} M_{\odot}\right)$ structures with sizes of $\sim 1-15$ pc (e.g., Carey et al. 1998; Simon et al. 2006b; Rathborne et al. 2006, hereafter RJS06; Du \& Yang 2008; Vasyunina et al. 2009). Most IRDCs are filamentary (e.g.,

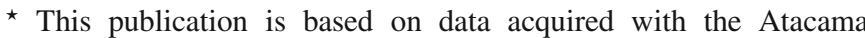
Pathfinder Experiment (APEX). APEX is a collaboration between the Max-Planck-Institut für Radioastronomie, the European Southern Observatory, and the Onsala Space Observatory.
Peretto \& Fuller 2009), and contain density enhancements, or clumps $^{1}$, that are visible in (sub)millimetre dust continuum maps (e.g., Carey et al. 2000; Garay et al. 2004; Ormel et al. 2005; RJS06). The cold clumps (i.e., clumps unassociated with MSX $8 \mu \mathrm{m}$ emission) identified by RJS06 have typical sizes and masses of $\sim 0.5 \mathrm{pc}$ and $\sim 120 M_{\odot}$, respectively. Because IRDCs have clumpy structures, they are likely to be in an early stage of fragmentation (e.g., RBG09 and references therein).

The radial galactocentric distribution of IRDCs peaks at $R_{\mathrm{GC}}=5 \mathrm{kpc}$ in the $1 \mathrm{st}$ Galactic quadrant, and at $R_{\mathrm{GC}}=$ $6 \mathrm{kpc}$ in the 4th quadrant, which correspond to the location of the Scutum-Centaurus spiral arm (see Simon et al. 2006b; and Jackson et al. 2008). This, together with the fact that IRDCs have sizes and masses similar to those of warm massive clusterforming regions (e.g., Lada \& Lada 2003; Motte et al. 2003), has led to the suggestion that IRDCs represent the very early stages of high-mass star/star cluster formation. Indeed, several studies have found signs of ongoing star formation within IRDCs. These

\footnotetext{
1 We prefer to use the term "clump" according to e.g., Ragan et al. (2009, hereafter RBG09), rather than the term "core" which was used by e.g., RJS06. Clumps have masses and sizes (radii) of $\sim 10-10^{3} M_{\odot}$ and $\sim 0.1-1$ pc, respectively (e.g., Bergin \& Tafalla 2007).
} 
include $\mathrm{CH}_{3} \mathrm{OH}$ and $\mathrm{H}_{2} \mathrm{O}$ masers (Beuther et al. 2002b; Pillai et al. 2006a; Wang et al. 2006; Ellingsen 2006), outflow signatures (Beuther et al. 2005; Beuther \& Sridharan 2007; Sakai et al. 2008), and associated infrared sources (e.g., Rathborne et al. 2005; Beuther \& Steinacker 2007; Chambers et al. 2009; RBG09). Cold, dense clumps are suggested to host/represent precursors of hot molecular cores, i.e., high-mass starless cores (HMSCs; e.g., Sridharan et al. 2005; Beuther et al. 2007). A few hot cores have been found within IRDCs (Rathborne et al. 2007, 2008), but it is important to increase the sample in order to study the sequence of events. It should be noted that the formation of hot cores may not be universal in IRDCs - some of them seem to form only low- to intermediate-mass stars (e.g., van der Wiel \& Shipman 2008).

\subsection{Infrared dark cloud MSXDC G304.74+1.32}

The IRDC studied in this paper was designated as MSXDC G304.74+1.32 (hereafter, G304.74) by Simon et al. (2006a). G304.74 was observed by Beltrán et al. (2006) with the SIMBA bolometer array on SEST at $1.2 \mathrm{~mm}$. Beltrán et al. (2006) identified eight millimetre clumps within the cloud, out of which four were found to be associated with MSX point sources. The distance to the cloud, $2.4 \mathrm{kpc}$, is a kinematic distance estimated from the velocity of the CS line observed by Fontani et al. (2005). The galactocentric distance is $\sim 7.4 \mathrm{kpc}$.

G304.74 was chosen for the present study because its relatively close distance allows for reasonably good spatial resolution in order to study its substructure. Moreover, a relatively high number of clumps (8) were already identified from the cloud (see above), and thus it was considered an appropriate object for studying IRDC fragmentation.

In this paper, we present the results of our $870 \mu \mathrm{m}$ dust continuum mapping of G304.74. The paper pays special attention to the clumpy structure of the cloud, and the clump mass distribution, and thereby addresses the fragmentation of IRDCs. The observations and data-reduction procedures are described in Sect. 2. The observational results are presented in Sect. 3. The MSX $8 \mu \mathrm{m}$ optical thicknesses toward the submm peaks are derived in Sect. 4. In Sect. 5, we describe the methods used to derive the physical properties of the observed clumps. In Sect. 6, we discuss the results of our study, and in Sect. 7, we summarise our main conclusions.

\section{Observations and data reduction}

\subsection{Submillimetre dust continuum}

The $870 \mu \mathrm{m}$ dust continuum observations toward G304.74 were carried out on 25-27 April 2009 with the 295 channel bolometer array LABOCA (Large APEX Bolometer Camera) on APEX. The central frequency of the instrument is $345 \mathrm{GHz}$, and the bandwidth is $60 \mathrm{GHz}$. The half-power beam width $(H P B W)$ of the telescope is $18^{\prime \prime} 6(\sim 0.22 \mathrm{pc}$ at $2.4 \mathrm{kpc})$ at the frequency used. The total field of view (FoV) of LABOCA is 11!4. The instrument and its observing modes are described in Siringo et al. (2009).

Absolute flux calibration was achieved through observations of the planets Mars, Uranus, and Neptune (the primary calibrators for LABOCA), and the star CW Leo as secondary calibrator. The uncertainty due to flux calibration was estimated to be $\sim 10 \%$. The telescope focus and pointing were checked using the planet Saturn, the star $\eta$ Carinae, and the $\mathrm{H}_{2} \mathrm{O}$ maser source B13134 (305.80-00.24). The submm zenith opacity was determined using the sky-dip method and the values varied from 0.20 to 0.38 , with a median value of 0.30 . The observations were thus conducted in fair weather conditions.

The observations were made using the on-the-fly (OTF) mapping mode with a scanning speed of $3^{\prime} \mathrm{s}^{-1}$. The size of the OTF map is $34^{\prime} \times 34^{\prime}$. This was achieved by 204 subscans of $34^{\prime}$ in length (parallel with the RA axis), spaced by $10^{\prime \prime}$. The area was mapped five times, with a total observing time of $5.2 \mathrm{~h}$. In this manner, a uniform rms noise level of $\sigma=0.03 \mathrm{Jy}$ beam $^{-1}$ was reached in the central $\sim 15^{\prime} \times 15^{\prime}$ area which covers the target source.

The data reduction was performed using the BoA (Bolometer Array Analysis Software) software package according to guidelines in the BoA User and Reference Manual (2007) ${ }^{2}$. The data reduction included flat-fielding, flagging bad/dark channels and data according to telescope speed and acceleration, correcting for the atmospheric opacity, division into subscans, baseline subtractions and median-noise removal (correction for sky noise), despiking, and filtering-out of the low frequencies of the $1 / f$-noise. Finally, the five individual maps were coadded.

We note that even the source model was iteratively used in the reduction process, the resulting final map (Fig. 1, left panel) has negative artefacts ("holes") around regions of bright emission. The depths of these negative holes are $\sim 15-50 \%(\sim 30 \%$ on average) of the nearest peak object brightness, and thus they are likely to introduce additional uncertainty in the source flux densities. We estimate that the total flux density uncertainty due to calibration and negative artefacts is in the range $\sim 20-50 \%$. These uncertainties are not, however, taken into account in the analyses presented in this paper.

\subsection{Complementary near-, mid-, and far-infrared data}

The target source $\left(l=304^{\circ} .74, b=1.32\right)$ is not included in the GLIMPSE $(3.6,4.5,5.8,8.0 \mu \mathrm{m})$ and MIPSGAL $(24$ and $70 \mu \mathrm{m})$ surveys of Spitzer which cover the Galactic latitudes $|b| \leq 1^{\circ}$. We have used the near-infrared 2MASS ( $J, H$, and $K_{\mathrm{s}}$ ) survey data archive (Skrutskie et al. 2006), data products from the MSX survey (Price et al. 2001; Egan et al. 2003), and the IRAS (Infrared Astronomical Satellite) MIR and far-infrared (FIR) observations.

\section{Observational results}

\subsection{Clump identification}

The obtained LABOCA map is presented in the left panel of Fig. 1. The cloud has a filamentary appearance and extends over about 13.2 , i.e., about 9.2 pc at the cloud's distance. In order to identify clumps in the LABOCA map, we employed the two-dimensional clumpfind algorithm, clfind2d, developed by Williams et al. (1994). The clfind2d routine determines the peak position, the $F W H M$ (full width at half maximum) size (not corrected for beam size), and the peak and total integrated flux density of clump based on specified contour levels. The algorithm requires two input parameters: 1) the intensity threshold, i.e., the lowest contour level, which determines the minimum emission to be included into clumps; and 2) the stepsize which determines the required "contrast" between two clumps to be considered as different objects. We set both the intensity threshold and the stepsize to $2 \sigma$ of the background noise level. With these settings, G304.74 divides into 12 clumps.

\footnotetext{
${ }^{2}$ http://www astro.uni-bonn.de/boawiki/Boa
} 

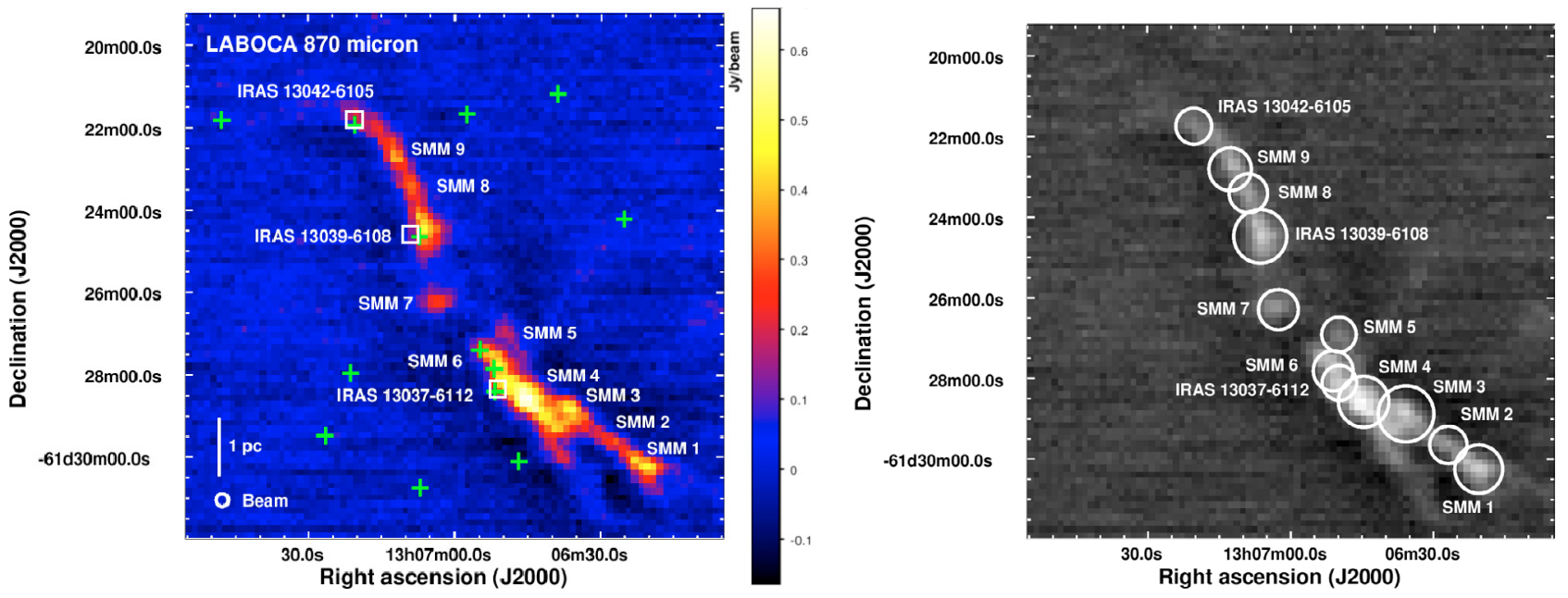

Fig. 1. Left: LABOCA map of the $870 \mu \mathrm{m}$ dust continuum emission from the IRDC G304.74. The green plus signs and white boxes mark the positions of the MSX $8 \mu \mathrm{m}$ sources in the field (cf. Fig. 2) and IRAS point sources, respectively. The intensity range is from -0.17 to $0.66 \mathrm{Jy}$ beam ${ }^{-1}$, as indicated in the colour bar. The beam HPBW (18'.6) and scale-bar are shown in the bottom left. Right: grey-scale image of $870 \mu$ m emission shown in the left panel with the locations and sizes of the submm clumps determined by the clumpfind algorithm. The circles represent the area associated with each clump but not the shape.

Table 1. Submillimetre clumps identified by the clumpfind algorithm in the IRDC G304.74.

\begin{tabular}{|c|c|c|c|c|c|c|c|}
\hline \multirow[b]{2}{*}{ Name } & \multicolumn{2}{|c|}{ Peak position } & \multirow{2}{*}{$\begin{array}{c}S_{870}^{\text {peak } a} \\
{\left[\mathrm{Jy} \mathrm{beam}^{-1} \text { ] }\right.}\end{array}$} & \multirow{2}{*}{$\begin{array}{r}S_{870}^{a} \\
{[\mathrm{Jy}]}\end{array}$} & \multirow{2}{*}{$\begin{array}{c}\theta_{\mathrm{s}} \\
{\left[{ }^{\prime \prime}\right]}\end{array}$} & \multirow{2}{*}{$\begin{array}{r}R_{\mathrm{eff}^{b}}^{b} \\
{\left[{ }^{\prime \prime}\right]}\end{array}$} & \multirow{2}{*}{$\begin{array}{l}1.2 \mathrm{~mm} \text { clump No. } \\
\text { (Beltrán et al. } 2006\end{array}$} \\
\hline & $\alpha_{2000.0}[\mathrm{~h}: \mathrm{m}: \mathrm{s}]$ & $\delta_{2000.0}\left[{ }^{\circ}:^{\prime}:^{\prime \prime}\right]$ & & & & & \\
\hline SMM 1 & 130620.5 & -613015 & 0.46 & 2.0 & 36 & 36 & \\
\hline SMM 2 & 130626.9 & -612939 & 0.30 & 1.0 & 38 & 28 & \\
\hline SMM 3 & 130635.8 & -612853 & 0.50 & 3.4 & 45 & 42 & 1 \\
\hline SMM 4 & 130644.7 & -612835 & 0.66 & 4.1 & 38 & 38 & 3 \\
\hline IRAS $13037-6112$ & 130649.8 & -612807 & 0.47 & 1.6 & 24 & 26 & 2 \\
\hline SMM 5 & 130649.9 & -612655 & 0.19 & 0.7 & 28 & 26 & \\
\hline SMM 6 & 130651.1 & -612749 & 0.46 & 1.8 & 27 & 30 & 4 \\
\hline SMM 7 & 130702.6 & -612618 & 0.26 & 0.9 & 24 & 30 & 8 \\
\hline IRAS 13039-6108 & 130706.4 & -612429 & 0.47 & 2.6 & 37 & 40 & 5 \\
\hline SMM 8 & 130708.9 & -612325 & 0.31 & 1.1 & 26 & 29 & \\
\hline SMM 9 & 130712.7 & -612249 & 0.38 & 1.6 & 35 & 32 & 6 \\
\hline IRAS $13042-6105$ & 130720.3 & -612145 & 0.21 & 0.8 & 28 & 27 & 7 \\
\hline
\end{tabular}

Notes. ${ }^{(a)}$ The peak and total flux densities have estimated uncertainties of $\sim 20-50 \%$, depending on the intensity of negative holes around the clump (see Sect. 2.1).

(b) The effective radius, $R_{\text {eff }}=\sqrt{A / \pi}$, where $A$ is the area assigned to the clump, i.e., area contained within a $2 \sigma$ contour. The radius has not been deconvolved from the beam size.

(c) Corresponding $1.2 \mathrm{~mm}$ clump in Beltrán et al. (2006).

We note that the total number and size of clumps identified by clumpfind is quite sensitive to the selected contour levels. The selected $2 \sigma$ contour levels turned out to give the best agreement with the identification by eye. This selection is also recommended by Williams et al. (1994). See also Pineda et al. (2009) for a recent discussion of clump identification with clumpfind. Pineda et al. (2009) concluded that small changes in the threshold and/or stepsize values can lead to important changes in the number of identified clumps.

\subsection{Observed properties of the clumps}

All the clumps have a peak flux density $>5 \sigma$ (i.e., $>0.15 \mathrm{Jybeam}^{-1}$ ) relative to the local background. The coordinates, peak and integrated flux densities, and deconvolved angular FWHM diameters $\left(\theta_{\mathrm{s}}\right)$ are listed in Cols. (2)-(6) of Table 1. The values of $\theta_{\mathrm{s}}$ correspond to the beam corrected $F W H M$ size. Column (7) lists the effective radius, $R_{\text {eff. }}$ This radius is defined as $R_{\text {eff }}=\sqrt{A / \pi}$, where $A$ is the projected area with the clump boundaries. The corresponding circles are overlaid on the LABOCA map in the right panel of Fig. 1. They are centred on the dust peak positions (Cols. (2) and (3)). Note that the clumpfind algorithm makes no assumption about the shape of the clump.

The left panel of Fig. 2 shows the wide-field MSX $8 \mu \mathrm{m}$ image (extracted from the MSX Galactic Plane Survey images ${ }^{3}$ ) towards G304.74. The MSX $8 \mu \mathrm{m}$ image zooming to the dark cloud with LABOCA contours is shown in the right panel of Fig. 2. There is a good agreement between the morphologies of the $870 \mu \mathrm{m}$ continuum emission and the $8 \mu \mathrm{m}$ extinction visible as a dark lane in the MSX image. The resolution of the MSX image, $18 . ' 3$, is similar to that of the $870 \mu \mathrm{m}$ LABOCA map.

Three of the submm clumps are associated with sources both from the MSX and IRAS point source catalogues (within $\sim 6-23^{\prime \prime}$ and $\sim 4-20^{\prime \prime}$ from the dust peak position,

\footnotetext{
${ }^{3}$ http://irsa.ipac.ipac.caltech.edu
} 

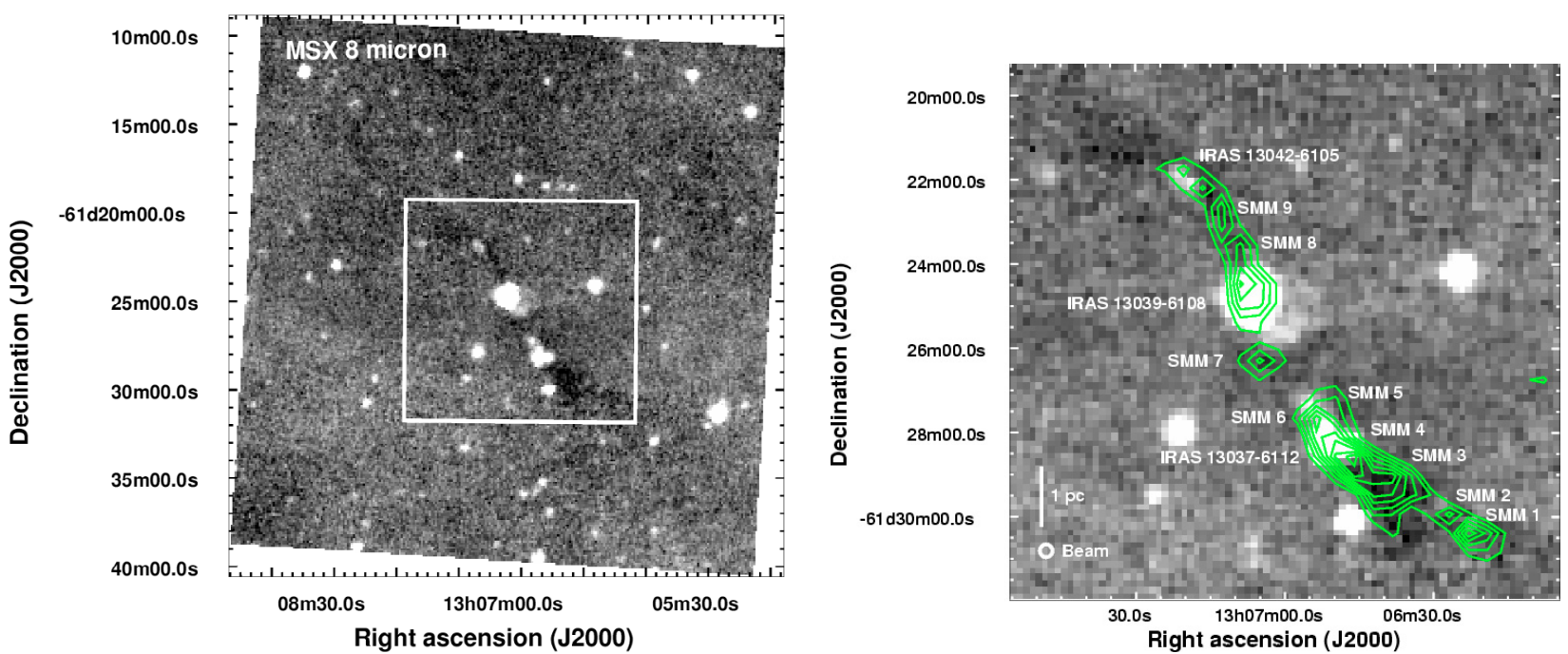

Fig. 2. Left: wide-field $(0.5 \times 0.5$, i.e., $\sim 21 \times 21 \mathrm{pc}$ at $2.4 \mathrm{kpc})$ MSX $8 \mu \mathrm{m}$ image showing the MIR extinction of the IRDC G304.74. Right: blow-up of the left panel showing the same region as in Fig. 1. The MSX $8 \mu \mathrm{m}$ image is overlaid with contours of LABOCA $870 \mu \mathrm{m}$ emission. The intensity range of a grey-scale image is from $1.86 \times 10^{-7}$ to $2.32 \times 10^{-5} \mathrm{~W} \mathrm{~m}^{-2} \mathrm{sr}^{-1}$. The contours are $0.06(2 \sigma)$ to $0.66 \mathrm{Jy}_{\text {beam }}^{-1}$, in steps of $0.06 \mathrm{Jy}^{\mathrm{beam}}{ }^{-1}$. The beam $H P B W\left(18^{\prime \prime} .3\right)$ and scale-bar are shown in the bottom left.

Table 2. MSX point sources in the IRDC G304.74.

\begin{tabular}{|c|c|c|c|c|c|}
\hline Associated clump & MSX6C designation & $\begin{array}{c}S_{8.28} \\
{[\mathrm{Jy}]}\end{array}$ & $\begin{array}{c}S_{12.13} \\
\text { [Jy] }\end{array}$ & $\begin{array}{c}S_{14.65} \\
\text { [Jy] }\end{array}$ & $\begin{array}{c}S_{21.34} \\
\text { [Jy] }\end{array}$ \\
\hline IRAS $13037-6112$ & G304.7728+01.3431 & $0.876 \pm 0.037$ & $<0.876 \pm 0.076$ & $1.027 \pm 0.076$ & $<1.969 \pm 0.142$ \\
\hline SMM 6 & G304.7738+01.3522 & $0.213 \pm 0.011$ & - & $<0.753 \pm 0.061$ & $2.972 \pm 0.193$ \\
\hline SMM 6 & G304.7800+01.3597 & $0.184 \pm 0.010$ & - & - & - \\
\hline IRAS 13039-6108 & G304.8074+01.4037 & $1.875 \pm 0.077$ & $2.125 \pm 0.123$ & $<0.730 \pm 0.063$ & $<1.678 \pm 0.129$ \\
\hline IRAS 13042-6105 & G304.8366+01.4472 & $0.147 \pm 0.010$ & - & - & - \\
\hline
\end{tabular}

respectively; see Fig. 1, left). These are designated according to the IRAS names. The remaining nine clumps are named SMM 1, SMM 2, etc. One of the submm clumps, SMM 6, appears to be associated with two $8 \mu \mathrm{m}$ sources from the MSX catalogue. The stronger $8 \mu \mathrm{m}$ source is located at $\sim 6^{\prime \prime}$ from the dust peak position $\left(0.46 \mathrm{Jy} \mathrm{beam}^{-1}\right)$, whereas the weaker one is located at $\sim 19^{\prime \prime}$ from the closest dust emission peak $\left(0.41 \mathrm{Jy} \mathrm{beam}^{-1}\right)$ within SMM 6. Furthermore, the eastern edge of SMM 4 is bright in $8 \mu \mathrm{m}$ as the MSX source associated with IRAS 13037-6112 extends about $55^{\prime \prime}$ west. Thus there are seven clumps which are completely dark in the MIR.

The flux densities at MIR and FIR wavelengths retrieved from the MSX $(8.28,12.13,14.65,21.34 \mu \mathrm{m})$ and IRAS $(12,25$, $60,100 \mu \mathrm{m})$ archives for all the IRAS sources and for the two $8 \mu \mathrm{m}$ point sources associated with SMM 6 are listed in Tables 2 and 3 , respectively.

\section{IRDC extinction and the $8 \mu \mathrm{m}$ optical thicknesses}

\subsection{Principle}

The observed $8 \mu \mathrm{m}$ intensity towards an IRDC, $I_{\mathrm{IRDC}}^{\mathrm{obs}}$, can be written as (e.g., Bacmann et al. 2000)

$I_{\mathrm{IRDC}}^{\mathrm{obs}}=I_{\mathrm{bg}} \mathrm{e}^{-\tau_{8} \mu \mathrm{m}}+I_{\text {fore }}$,

where $I_{\mathrm{bg}}$ is the $8 \mu \mathrm{m}$ intensity of the background emission, $\tau_{8} \mu \mathrm{m}$ is the $8 \mu \mathrm{m}$ optical thickness, and $I_{\text {fore }}$ is the $8 \mu \mathrm{m}$ intensity
Table 3. IRAS point sources in the IRDC G304.74.

\begin{tabular}{|c|c|c|c|c|}
\hline Name & $\begin{array}{l}S_{12} \\
{[\mathrm{Jy}]}\end{array}$ & $\begin{array}{l}S_{25} \\
{[\mathrm{Jy}]}\end{array}$ & $\begin{array}{c}S_{60} \\
{[\mathrm{Jy}]} \\
\end{array}$ & $\begin{array}{l}S_{100} \\
{[\mathrm{Jy}]}\end{array}$ \\
\hline IRAS 13037-6112 & 1.84 & 7.41 & 64.96 & $<196.5$ \\
\hline IRAS 13039-6108 & 3.99 & 7.43 & 105.6 & 196.5 \\
\hline IRAS 13042-6105 & $<1.31$ & 0.67 & $<4.97$ & $<196.5$ \\
\hline
\end{tabular}

contribution from foreground material. Equation (1) can be inverted to get the value of $\tau_{8} \mu \mathrm{m}$ as

$\tau_{8 \mu \mathrm{m}}=-\ln \left(\frac{I_{\mathrm{IRDC}}^{\mathrm{obs}}-I_{\text {fore }}}{I_{\mathrm{bg}}}\right)$.

Following the notation of Bacmann et al. (2000) and Peretto \& Fuller (2009), the observed MIR intensity around the IRDC is

$I_{\mathrm{MIR}}=I_{\mathrm{bg}}+I_{\text {fore }}$.

To obtain an estimate for the value of $I_{\mathrm{MIR}}$, we used the technique of spatial median filtering (Simon et al. 2006a). In this method, $I_{\text {MIR }}$ for each pixel is estimated by taking the median of intensities inside a surrounding filter region. The size of the filter must be larger than the size of IRDC; the method will only capture emission fluctuations on scales larger than the size of the cloud. Given the size of G304.74 ( $\gtrsim 13^{\prime}$, i.e., over $1306^{\prime \prime}$ pixels of the MSX map), we used a circular spatial filter with a radius of $15^{\prime}$ (the same as used by Simon et al. 2006a). We note that the total size of the image used in the filtering process was $0.5 \times 0.5$ (see Fig. 2, left). Figure 3 shows the obtained model of $I_{\text {MIR }}$. Both the 


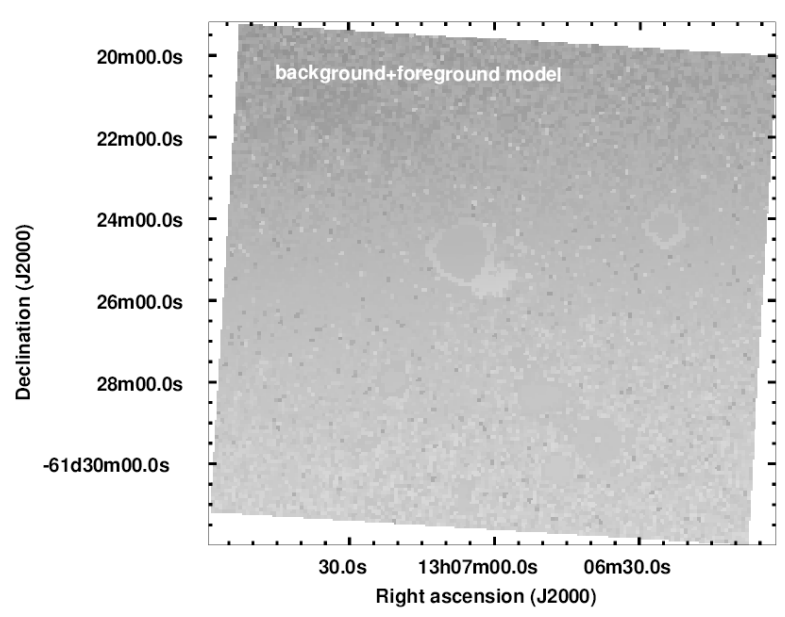

Fig. 3. Median filter estimate of $I_{\text {MIR }}$. The intensity scale is linear from $1.20 \times 10^{-6}$ to $1.26 \times 10^{-6} \mathrm{~W} \mathrm{~m}^{-2} \mathrm{sr}^{-1}$, with both mean and median being $1.23 \pm 0.02 \times 10^{-6} \mathrm{~W} \mathrm{~m}^{-2} \mathrm{sr}^{-1}$.

mean and median value of $I_{\text {MIR }}$ are $1.23 \pm 0.02 \times 10^{-6} \mathrm{~W} \mathrm{~m}^{-2} \mathrm{sr}^{-1}$, where the uncertainty is the standard deviation of the pixel values. The above value was adopted in the following analysis.

\subsection{Background and foreground estimation}

To estimate the contribution of $I_{\mathrm{bg}}$ and $I_{\text {fore }}$ to the value of $I_{\mathrm{MIR}}$ (Eq. (3), and Fig. 3), we used the similar analysis as used by Butler \& Tan (2009, their Sect. 3.1). We assumed that the radial distribution of Galactic $8 \mu \mathrm{m}$ PAH (polycyclic aromatic hydrocarbon) emission has the same exponential form as the radial distribution of molecular gas surface density in the Galaxy (the radial scale length is $H_{\mathrm{R}}=3.5 \pm 1.0 \mathrm{kpc}$; Williams \& McKee 1997). We note that this distribution is similar to that of the Galactic surface density of OB associations (McKee \& Williams 1997), which was used by Butler \& Tan (2009, their Eq. (3)). The assumption that the radial distribution of PAHs follows the molecular gas is supported by the results of Mattila et al. (1999) who found that the UIR (unidentified infrared) emission band intensities in the disk of the galaxy NGC 891 closely follow those of the $\mathrm{CO}$ emission and $1.3 \mathrm{~mm}$ dust continuum emission. However, UIR band intensities were found to be quite different from that of the $\mathrm{H} \alpha$ emission (see Fig. 5 of Mattila et al. 1999). Moreover, Kahanpää et al. (2003) found strong correlation between the Galactic UIR bands and $\mathrm{CO}$ emission. To estimate the vertical distribution of PAH emission, we used the Galactic vertical distribution of $\mathrm{CO}$ emission and adopted the scale length $z_{1 / 2}=70 \pm 10$ pc (Dame et al. 1987; Bronfman et al. 1988; Malhotra 1994).

We first calculated the so-called "foreground intensity ratio", $f_{\text {fore }}$, which is defined as the ratio of the column of PAH emission between the observer and the cloud and the total column through the Galaxy along the same line of sight, up to the galactocentric distance $16 \mathrm{kpc}$. For G304.74, we obtained $f_{\text {fore }}=0.514 \pm 0.05$, where the uncertainty represents the mimimum-maximum error based on the uncertainties in the scale lengths (see above). The distance of the Sun from the Galactic Centre was assumed to be $8.5 \mathrm{kpc}$ in this calculation. The intensity of the background emission is then given by

$I_{\mathrm{bg}}=\left(1-f_{\text {fore }}\right) I_{\mathrm{MIR}}$

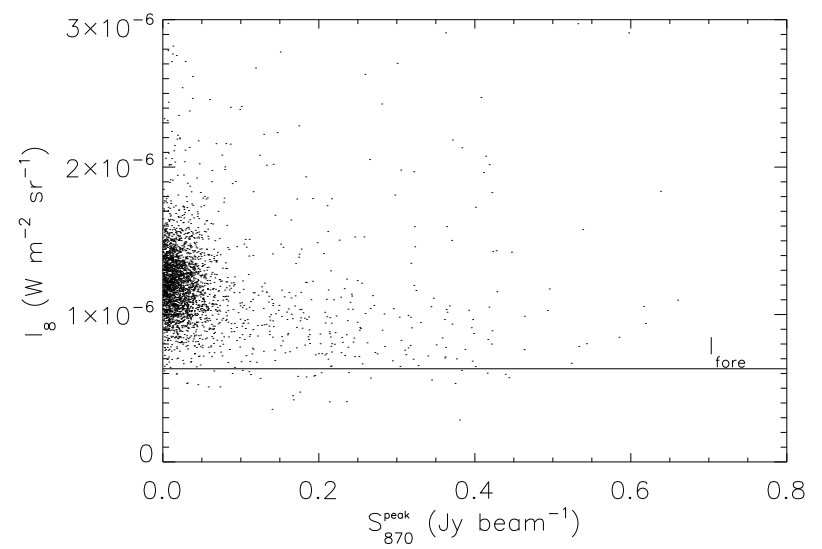

Fig. 4. MSX $8 \mu \mathrm{m}$ versus LABOCA $870 \mu \mathrm{m}$ intensity for G304.74. The horizontal line marks the $8 \mu \mathrm{m}$ foreground intensity, $I_{\text {fore }}=6.32 \times$ $10^{-7} \mathrm{~W} \mathrm{~m}^{-2} \mathrm{sr}^{-1}$, derived from the foreground intensity ratio, $f_{\text {fore }}$ (see main text).

The resulting values are $I_{\mathrm{bg}}=5.98 \pm 0.62 \times 10^{-7} \mathrm{~W} \mathrm{~m}^{-2} \mathrm{sr}^{-1}$ and $I_{\text {fore }}=6.32 \pm 0.65 \times 10^{-7} \mathrm{~W} \mathrm{~m}^{-2} \mathrm{sr}^{-1}$. The errors in $I_{\mathrm{bg}}$ and $I_{\text {fore }}$ are propagated from the standard deviation of $I_{\text {MIR }}$ and the uncertainty in $f_{\text {fore }}$. The above value of $I_{\text {fore }}$ is in good agreement with the lowest observed $8 \mu \mathrm{m}$ intensities as demonstrated in Fig. 4. We note that the anti-correlation between the submm and MIR intensities allows for a determination of $I_{\mathrm{bg}}$ and $I_{\mathrm{fore}}$ (e.g., Johnstone et al. 2003, Fig. 3 therein). However, the correlation shown in Fig. 4 is not very clear and thus only the $f_{\text {fore }}$-method is considered in the present paper (see Sect. 6.1 for further discussion).

The observed $8 \mu \mathrm{m}$ intensities toward the submm dust continuum peak positions, and the $8 \mu \mathrm{m}$ optical thicknesses calculated from Eq. (2), are listed in Cols. (2) and (3) of Table 4. The formal error in $\tau_{8 \mu \mathrm{m}}^{\text {peak }}$ was calculated by propagating the errors in $I_{\text {fore }}$ and $I_{\mathrm{bg}}$. In order to determine $I_{\mathrm{IRDC}}^{\mathrm{obs}}$ values in the submm peak positions, the MSX image was gridded with 9.'3 pixels, i.e., the LABOCA pixel size. We also estimated the possibility that part of the observed $8 \mu \mathrm{m}$ intensity originates from the bright surroundings of a MIR dark clump. The measured point spread function (PSF) in the MSX image is a Gaussian with a $F W H M$ of $22^{\prime \prime}$. Convolution with this PSF reduces the breadths of dark filaments, and, for the narrowest of them, increases the minimum intensity in the middle. We estimate that for a completely opaque source with a diameter of $\$ 30^{\prime \prime}$, the MIR radiation "leakage" from the surroundings amounts to $\gtrsim 20 \%$ of the background level. This contribution was, however, neglected in the above analysis which uses only the peak (minimum) $8 \mu \mathrm{m}$ intensities. Also, most of the MIR dark clumps are larger than $30^{\prime \prime}$ (see Table 1, Cols. (6) and (7)).

\subsection{Dust temperature estimates}

Because dust continuum emission is optically thin at (sub)mm wavelengths, the $870 \mu \mathrm{m}$ radiation intensity is given by

$I_{870} \simeq B_{870}\left(T_{\mathrm{d}}\right) \tau_{870}$

where $B_{870}\left(T_{\mathrm{d}}\right)$ is the Planck function with dust temperature $T_{\mathrm{d}}$. According to the Ossenkopf \& Henning (1994, hereafter OH94) dust model used in the present paper (see Sect. 5.1 for more 
Table 4. The observed $8 \mu \mathrm{m}$ intensity, the peak optical thickness, and the dust temperature toward the submm peak positions.

\begin{tabular}{cccc}
\hline \hline & $\begin{array}{c}I_{\mathrm{IRDC}}^{\text {obs }} \\
{\left[10^{-7} \mathrm{~W} \mathrm{~m}^{-2} \mathrm{sr}^{-1}\right]}\end{array}$ & $\tau_{8 \mu \mathrm{m}}^{\text {peak }}$ & $\begin{array}{c}T_{\mathrm{d}} \\
{[\mathrm{K}]}\end{array}$ \\
Name & 9.89 & $0.52 \pm 0.21$ & $30.6 \pm 17.0$ \\
\hline SMM 1 & 10.39 & $0.38 \pm 0.19$ & $28.0 \pm 19.6$ \\
SMM 2 & 11.73 & $0.10^{a}$ & $-{ }^{b}$ \\
SMM 3 & 10.97 & $0.25 \pm 0.17$ & $-^{b}$ \\
SMM 4 & 8.03 & $1.25 \pm 0.40$ & $10.1 \pm 2.8$ \\
SMM 5 & 8.08 & $1.22 \pm 0.39$ & $12.0 \pm 3.7$ \\
SMM 7 & 7.58 & $1.56 \pm 0.53$ & $11.5 \pm 3.7$ \\
SMM 8 & 6.99 & $2.19 \pm 0.98$ & $10.8 \pm 4.3$ \\
SMM 9 & & & \\
\hline
\end{tabular}

Notes. ${ }^{(a)}$ No error given because it is greater than the actual value. (b) The value of $T_{\mathrm{d}}$ could not be reasonably estimated.

details), the ratio of dust opacities per unit dust mass at $8.8^{4}$ and $870 \mu \mathrm{m}, \kappa_{8} / \kappa_{870}$, and thus the correponding ratio of the optical thicknesses, $\tau_{8} / \tau_{870}$, is about 865 . The obtained ratio is consistent with the results of Johnstone et al. (2003) and Ormel et al. (2005), who found that (assuming $T_{\mathrm{d}}=15 \mathrm{~K}$ ) $\kappa_{8} / \kappa_{850} \sim 640$ and 870 , respectively. By using this ratio, and the $\tau_{8}$ values derived above, estimates for the dust temperatures, $T_{\mathrm{d}}$, towards the submm peaks can be derived from Eq. (5). The resulting values are listed in Col. (4) or Table 4. The quoted errors are the minimum-maximum errors derived from the uncertainty in $\tau_{8}$.

The uncertainties of the $T_{\mathrm{d}}$ estimates are very large in the southern part (SMM 1, SMM 2; in the case of SMM 3 and SMM 4 no reasonable estimate could be obtained because of the large error in $\tau_{8}$ ). On the other hand, the estimates with moderate formal errors in the central and northern part of the cloud suggest low temperatures of slightly above $10 \mathrm{~K}$. It should be noted, however, that there are large uncertainties concerning the dust opacities and the determination of the $\tau_{8}$. This issue will be raised again in Sect. 6.2.

In view of these uncertainties, dust temperatures estimated above should be compared with previous temperature determinations from molecular lines. Observations towards several other IRDC clumps $\left(\mathrm{H}_{2} \mathrm{CO}\right.$, Carey et al. 1998; $\mathrm{CH}_{3} \mathrm{CCH}$, Teyssier et al. 2002; $\mathrm{NH}_{3}$, Pillai et al. 2006b; Sakai et al. 2008) provide gas kinetic temperatures of $T_{\text {kin }} \approx 10-20 \mathrm{~K}$. The assumption that $T_{\mathrm{d}}=T_{\text {kin }}$ is probably valid in dense clouds $\left(n\left(\mathrm{H}_{2}\right) \gtrsim 10^{5} \mathrm{~cm}^{-3}\right.$; e.g., Goldsmith \& Langer 1978). Based on these considerations, we assume in what follows that in general $T_{\mathrm{d}}=15 \mathrm{~K}$. Exceptions are made in the cases of two IRAS sources, for which temperatures can be derived from the spectral energy distributions (SEDs). We will also separately consider the possibility that $T_{\mathrm{d}}$ is elevated in the four southern clumps SMM 1-4.

\section{Physical parameters of the clumps}

\subsection{Spectral energy distributions of the IRAS sources}

The MSX, IRAS, LABOCA, and SIMBA data were used to fit the spectral energy distributions (SEDs) of IRAS 13037-6112 and IRAS 13039-6108. The SIMBA $1.2 \mathrm{~mm}$ flux densities of these sources are 0.89 and $1.36 \mathrm{Jy}$, respectively (Beltrán et al. 2006; Table 2 therein). Note that there is not enough data points for IRAS 13042-6105 in order to construct a reasonable SED

\footnotetext{
${ }^{4}$ Note, for the MSX band A filter the 50\% cutoffs are at 6.8 and $10.8 \mu \mathrm{m}$ (central wavelength is $8.8 \mu \mathrm{m}$ ), whereas the isophotal central wavelength of the filter is $8.28 \mu \mathrm{m}$.
}
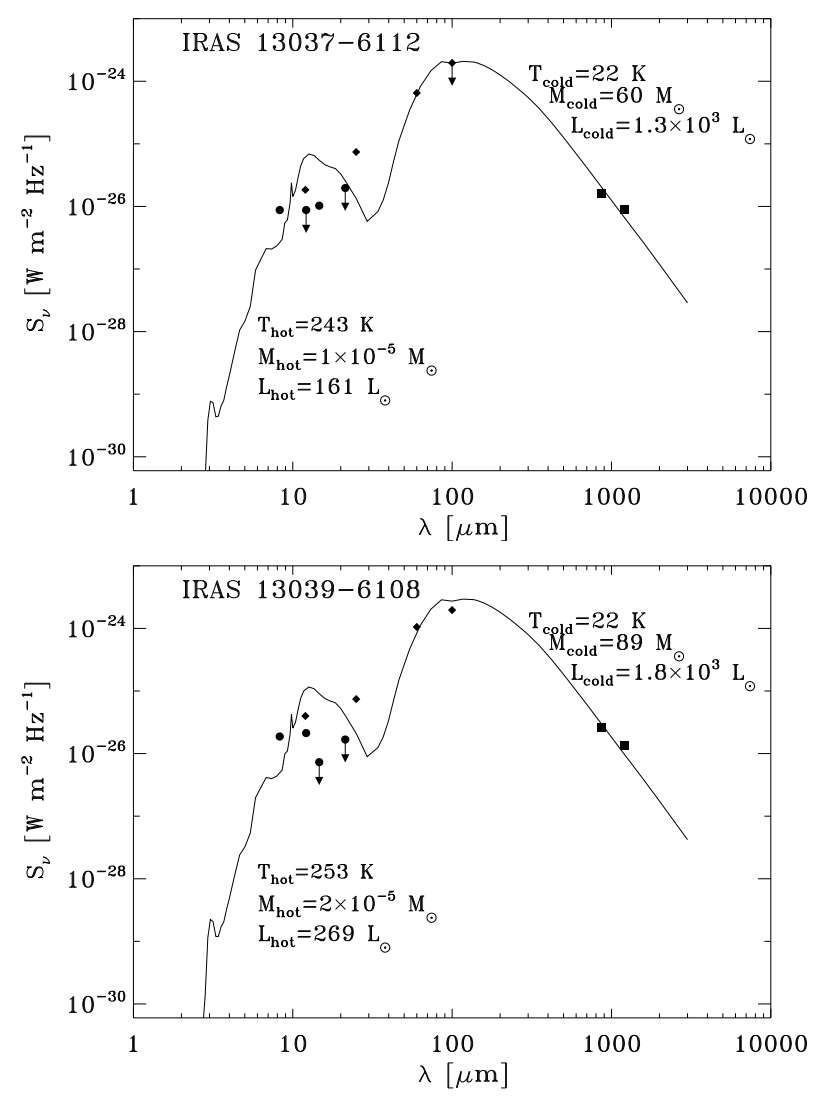

Fig. 5. Best-fit SEDs of IRAS 13037-6112 and IRAS 13039-6108. MSX data points are shown by circles, IRAS data points are shown by diamonds, and LABOCA and SIMBA (sub)mm data points are indicated by squares. Upper limits are indicated by arrows. The solid lines in both plots represent the sum of two (cold+hot) components (see Cols. (4) and (5) of Table 5). The absorption features appearing at $\sim 3.1$ and $10 \mu \mathrm{m}$ are attributed to the $\mathrm{H}_{2} \mathrm{O}$ ice and silicates, respectively (OH94 and references therein).

(e.g., most of its IRAS flux densities are only upper limits, see Table 3). The derived SEDs are shown in Fig. 5. The leastsquares fitting routine used in the derivation of the $\mathrm{SED}^{5} \mathrm{~min}-$ imises the $\operatorname{sum} \sum_{i=1}^{N}\left[\log _{10}\left(S_{v}^{\text {obs }}\left(\lambda_{i}\right)\right)-\log _{10}\left(S_{v}^{\bmod }\left(\lambda_{i}\right)\right)\right]^{2}$, where $N$ is the number of data points (7 and 8 for IRAS 13037-6112 and 13039-6108, respectively), $S_{v}^{\text {obs }}\left(\lambda_{i}\right)$ is the observed flux density, and $S_{v}^{\bmod }\left(\lambda_{i}\right)$ is the model flux density. In both cases, the data were fitted by a two-temperature composite model. It was assumed that both components at different temperatures emit as a blackbody modified by the wavelength-dependent dust opacity, $\kappa_{\lambda}$ (see below). The best-fit model SEDs overestimate the flux densities at $\sim 12-20 \mu \mathrm{m}$, but underestimate them at $\sim 8$ and $25 \mu \mathrm{m}$. It should be noted that the flux densities included in the SEDs are measured using telescopes with different beam sizes. Thus the flux densities obtained for extended sources are not fully comparable, and this can in part explain discrepancies between MSX (18'.3) and IRAS ( 2 $2^{\prime}$ at $12 \mu \mathrm{m}$ to $\sim 4^{\prime}$ at $100 \mu \mathrm{m}$ ) fluxes at 12 and $\sim 20-25 \mu \mathrm{m}$. On the other hand, SIMBA and LABOCA flux densities at $\lambda=1.2 \mathrm{~mm}$ and $0.87 \mathrm{~mm}$ refer to clump areas ( $R_{\mathrm{eff}}$ is typically $\left.\sim 30^{\prime \prime}\right)$ derived by clumpfind. $R_{\text {eff }}$ values are similar for both IRAS $13037-$ 6122 and IRAS 13039-6108 ( 30-40" or $\sim 0.35-0.47 \mathrm{pc})$. Assuming that the emission in the IRAS bands is confined in

\footnotetext{
5 The SED fitting routine was originally written by J. Steinacker.
} 
Table 5. Results of the SED fits.

\begin{tabular}{cccccccc}
\hline \hline & $M_{\text {tot }}$ & $L_{\text {bol }}$ & $T_{\text {cold }}$ & $T_{\text {hot }}$ & & & $M_{\text {tot }} / L_{\text {bol }}$ \\
Source & {$\left[M_{\odot}\right]$} & {$\left[10^{3} L_{\odot}\right]$} & {$[\mathrm{K}]$} & {$[\mathrm{K}]$} & $M_{\text {cold }} / M_{\text {tot }}$ & $L_{\text {cold }} / L_{\text {bol }}$ & {$\left[M_{\odot} / L_{\odot}\right]$} \\
\hline IRAS 13037-6112 & 60 & 1.5 & 22 & 243 & $\sim 1$ & 0.90 & 0.04 \\
IRAS 13039-6108 & 89 & 2.1 & 22 & 253 & $\sim 1$ & 0.87 & 0.04 \\
\hline
\end{tabular}

the region of the submm clump, the characteristic spatial scale associated with the SEDs is $\lesssim 0.5 \mathrm{pc}$.

We have adopted a dust-to-gas mass ratio of $R_{\mathrm{d}}=1 / 100$, a value which has often been used in the IRDC studies (e.g., RJS06; Vasyunina et al. 2009; Parsons et al. 2009). However, this value can differ from $1 / 100$. For instance, Draine et al. (2007) determined a value of $R_{\mathrm{d}} \approx 1 / 186$ based on observed depletions of heavy elements in the Galaxy. Dust opacities we have adopted correspond to a MRN size distribution with thick ice mantles ${ }^{6}$ at a gas density of $n_{\mathrm{H}}=10^{5} \mathrm{~cm}^{-3}$ (OH94). The resulting SED parameters are given in Table 5. The total (cold+hot) mass and the integrated bolometric luminosity are given in Cols. (2) and (3) of Table 5, respectively. The temperatures of the two components are listed in Cols. (4) and (5). In Cols. (6) and (7), we give the mass and luminosity fractions of the cold component versus the total mass and luminosity. Column (8) lists the massto-luminosity ratio, $M_{\mathrm{tot}} / L_{\mathrm{bol}}$, which is an evolutionary indicator of the clump as it is expected to decrease with time. The envelope mass decreases during the star formation process, and the luminosity of the embedded star or stellar cluster rises (e.g., Sridharan et al. 2002). We note that the adopted dust opacity model (corresponding to particles coated with thick ice mantles) is not likely to be appropriate for hot dust, and therefore the total bolometric luminosity for the hot component should be taken with caution. The MIR spectral features (such as PAH emission) also cause the fit to the hot component being more uncertain than the fit to the cold part of the spectrum. However, this should not alter the fact that for both IRAS sources the mass of the hot component is negligible $\left(\sim 1-2 \times 10^{-5} M_{\odot}\right)$, and thus the bulk of the material is cold ( $\left.M_{\text {cold }} / M_{\text {tot }} \sim 1\right)$. The bolometric temperature of IRAS $13039-6108$ ( $T_{\text {cold }} \simeq 22 \mathrm{~K}$ ) is in good agreement with the rotation temperature of $18 \mathrm{~K}$ derived from $\mathrm{C}^{17} \mathrm{O}$ by Fontani et al. (2005).

\subsection{Linear sizes, masses, and $\mathrm{H}_{2}$ number densities}

The clump linear radii were computed from the effective radii listed in Col. (7) of Table 1. The clump masses (gas+dust mass, $M_{\text {cont }}$ ) were calculated from their integrated $870 \mu \mathrm{m}$ flux density, $S_{870}$ (Table 1, Col. (5)), assuming that the thermal dust emission is optically thin (Hildebrand 1983):

$M_{\text {cont }}=\frac{S_{870} d^{2}}{B_{870}\left(T_{\mathrm{d}}\right) K_{870} R_{\mathrm{d}}}$,

where $d$ is the source distance. For IRAS 13037-6112 and 13039-6108, we assumed the dust temperatures to be the same as their bolometric temperatures, $T_{\text {cold }} \simeq 22 \mathrm{~K}$, resulting from SED

\footnotetext{
${ }^{6}$ In cold, dense interiors of the IRDCs, dust grains are supposed to be coagulated and covered by icy mantles (e.g., Peretto \& Fuller 2009, and references therein). This is supported by e.g., the observed depletion of $\mathrm{H}_{2} \mathrm{CO}$ (Carey et al. 1998, 2000), CO (Pillai et al. 2007; Zhang et al. 2009), and CS (Beuther \& Henning 2009) in IRDCs. Moreover, Butler \& Tan (2009) found some evidence for dust opacity changes within IRDCs, which could be caused by ice mantle formation and grain growth.
}

fits (see Table 5, Col. (4)). For all the other clumps (SMM 1, SMM $2, \ldots$, and IRAS 13042-6105), it was assumed that $T_{\mathrm{d}}=$ $15 \mathrm{~K}$ (Sect. 4.3). We assumed that $\kappa_{870} \simeq 0.17 \mathrm{~m}^{2} \mathrm{~kg}^{-1}$. This value is interpolated from $\mathrm{OH} 94$, see Sect. 5.1. As in the SED fits, the value $1 / 100$ is adopted for $R_{\mathrm{d}}$.

The volume-averaged $\mathrm{H}_{2}$ number densities, $\left\langle n\left(\mathrm{H}_{2}\right)\right\rangle$, were calculated assuming a spherical geometry for the clumps, using the formula

$\left\langle n\left(\mathrm{H}_{2}\right)\right\rangle=\frac{\langle\rho\rangle}{\mu_{\mathrm{H}_{2}} m_{\mathrm{H}}}$,

where $\langle\rho\rangle=M_{\text {cont }} /\left(4 / 3 \pi R_{\text {eff }}^{3}\right)$ is the matter density, $\mu_{\mathrm{H}_{2}}=2.8$ is the mean molecular weight per $\mathrm{H}_{2}$ molecule (assuming a $10 \%$ helium abundance), and $m_{\mathrm{H}}$ is the mass of the hydrogen atom. The obtained radii, masses, and volume-averaged $\mathrm{H}_{2}$ number densities are given in Cols. (2), (3), and (7) of Table 6, respectively. The typical density in the clumps is likely to be higher than the volume-averaged value because of substructure. Note that our masses are in general larger than those derived by Beltrán et al. (2006) who assumed a dust temperature of $30 \mathrm{~K}$ and a $\kappa_{1.2 \mathrm{~mm}}$ of $0.1 \mathrm{~m}^{2} \mathrm{~kg}^{-1}$ in their mass estimates. They also obtained higher densities because of using the $F W H M$ radii (instead of effective radii) and a mean molecular weight of 2.29.

\section{3. $\mathrm{H}_{2}$ column densities}

The $\mathrm{H}_{2}$ column densities, $N\left(\mathrm{H}_{2}\right)$, towards the submm peaks were estimated using 1) the dust emission at $870 \mu \mathrm{m}$, and 2) the dust extinction at $8 \mu \mathrm{m}$. In the first method, the LABOCA intensities were converted to $\mathrm{N}\left(\mathrm{H}_{2}\right)$ using the equation

$N_{870}\left(\mathrm{H}_{2}\right)=\frac{I_{870}^{\text {dust }}}{B_{870}\left(T_{\mathrm{d}}\right) \mu_{\mathrm{H}_{2}} m_{\mathrm{H}} \kappa_{870} R_{\mathrm{d}}}$,

where $I_{870}^{\text {dust }}=S_{870}^{\text {peak }} / \Omega_{\text {beam }}$ is the observed dust peak surface brightness $\left(\Omega_{\text {beam }}\right.$ is the beam solid angle), which is related to the peak flux density via $1 \mathrm{Jy} / 18^{\prime \prime}$. 6 beam $=1.085 \times$ $10^{-18} \mathrm{~W} \mathrm{~m}^{-2} \mathrm{~Hz}^{-1} \mathrm{sr}^{-1}$. The dust temperature values used were identical to those adopted in the mass estimates (Eq. (6)). The uncertainty in $N_{870}\left(\mathrm{H}_{2}\right)$ was calculated by propagating the uncertainty in $I_{870}^{\text {dust }}$, and thus reflects only the $1 \sigma$ rms noise in the $870 \mu \mathrm{m}$ map.

The MSX $8 \mu$ m optical thicknesses (Table 4, Col. (3)) were used to estimate the peak $\mathrm{H}_{2}$ column densities by applying the formula

$N_{8}\left(\mathrm{H}_{2}\right)=\frac{\tau_{8 \mu \mathrm{m}}^{\text {peak }}}{\sigma_{\lambda}}$

where $\sigma_{\lambda}$ is the dust extinction cross-section per $\mathrm{H}_{2}$ molecule. According to the $\mathrm{OH} 94$ dust model we have used (see Sect. 5.1), the value $\sigma_{\lambda}=\kappa_{\lambda} \mu_{\mathrm{H}_{2}} m_{\mathrm{H}} R_{\mathrm{d}} \approx 7.0 \times 10^{-23} \mathrm{~cm}^{2}$ at $8.8 \mu \mathrm{m}$. The uncertainty in $N_{8}\left(\mathrm{H}_{2}\right)$ was calculated by propagating the uncertainty in $\tau_{8 \mu \mathrm{m}}^{\text {peak }}$ and do not include the systematic error resulting from the estimate of $\sigma_{\lambda}$. The obtained $\mathrm{H}_{2}$ column densities, and the $N_{870} / N_{8}$ ratios, are given in Cols. (4)-(6) of Table 6. 
A\&A 520, A102 (2010)

Table 6. Linear radii, masses, and $\mathrm{H}_{2}$ column and volume-averaged number densities of the submm clumps within the IRDC G304.74.

\begin{tabular}{ccccccc}
\hline \hline & $\begin{array}{c}R_{\text {eff }} \\
{[\mathrm{pc}]}\end{array}$ & $\begin{array}{c}M_{\text {cont }} \\
{\left[M_{\odot}\right]}\end{array}$ & $\begin{array}{c}N_{870}\left(\mathrm{H}_{2}\right) \\
{\left[10^{22} \mathrm{~cm}^{-2}\right]}\end{array}$ & $\begin{array}{c}N_{8}\left(\mathrm{H}_{2}\right) \\
{\left[10^{22} \mathrm{~cm}^{-2}\right]}\end{array}$ & $\begin{array}{c}N_{870} / N_{8} \\
\text { Source }\end{array}$ & $\begin{array}{c}\left\langle n\left(\mathrm{H}_{2}\right)\right\rangle \\
{\left[10^{4} \mathrm{~cm}^{-3}\right]}\end{array}$ \\
\hline SMM 1 $^{a}$ & 107 & $2.06 \pm 0.13$ & $0.74 \pm 0.30$ & $2.8 \pm 1.1$ & 0.7 \\
SMM 2 $^{b}$ & 0.33 & 53 & $1.35 \pm 0.14$ & $0.54 \pm 0.27$ & $2.5 \pm 1.3$ & 0.7 \\
SMM 3 $^{c}$ & 0.49 & 182 & $2.24 \pm 0.13$ & $0.14^{d}$ & $16 \pm 0.9$ & 0.7 \\
SMM 4 $^{e}$ & 0.44 & 219 & $2.96 \pm 0.13$ & $0.36 \pm 0.24$ & $8.2 \pm 5.5$ & 1.2 \\
IRAS 13037-6112 $^{2}$ & 0.30 & 48 & $1.17 \pm 0.07$ & - & - & 0.8 \\
SMM 5 & 0.30 & 37 & $0.85 \pm 0.13$ & $1.79 \pm 0.57$ & $0.5 \pm 0.2$ & 0.6 \\
SMM 6 & 0.35 & 96 & $2.06 \pm 0.13$ & - & - & 1.0 \\
SMM 7 & 0.35 & 48 & $1.17 \pm 0.14$ & $1.74 \pm 0.56$ & $0.7 \pm 0.2$ & 0.5 \\
IRAS 13039-6108 & 0.47 & 78 & $1.18 \pm 0.08$ & - & - & 0.3 \\
SMM 8 & 0.34 & 59 & $1.39 \pm 0.13$ & $2.23 \pm 0.76$ & $0.6 \pm 0.2$ & 0.7 \\
SMM 9 & 0.37 & 85 & $1.70 \pm 0.13$ & $3.13 \pm 1.40$ & $0.5 \pm 0.2$ & 0.8 \\
IRAS 13042-6105 & 0.31 & 43 & $0.94 \pm 0.13$ & - & - & 0.7 \\
\hline
\end{tabular}

Notes. ${ }^{(a)}$ By assuming $T_{\mathrm{d}}=30 \mathrm{~K}$ (see Sect. 4.3 and Table 4), $M_{\text {cont }}=39 M_{\odot}, N_{870}\left(\mathrm{H}_{2}\right)=0.75 \pm 0.05 \times 10^{22} \mathrm{~cm}^{-2}, N_{870} / N_{8}=1.0 \pm 0.1$, and $\left\langle n\left(\mathrm{H}_{2}\right)\right\rangle=0.2 \times 10^{4} \mathrm{~cm}^{-3}$.

(b) By assuming $T_{\mathrm{d}}=30 \mathrm{~K}, M_{\text {cont }}=20 M_{\odot}, N_{870}\left(\mathrm{H}_{2}\right)=0.49 \pm 0.05 \times 10^{22} \mathrm{~cm}^{-2}, N_{870} / N_{8}=0.9 \pm 0.1$, and $\left\langle n\left(\mathrm{H}_{2}\right)\right\rangle=0.3 \times 10^{4} \mathrm{~cm}^{-3}$.

(c) By assuming $T_{\mathrm{d}}=30 \mathrm{~K}, M_{\text {cont }}=66 M_{\odot}, N_{870}\left(\mathrm{H}_{2}\right)=0.82 \pm 0.05 \times 10^{22} \mathrm{~cm}^{-2}, N_{870} / N_{8}=5.9 \pm 0.4$, and $\left\langle n\left(\mathrm{H}_{2}\right)\right\rangle=0.3 \times 10^{4} \mathrm{~cm}^{-3}$.

(d) No error given because it is greater than the actual value.

(e) By assuming $T_{\mathrm{d}}=30 \mathrm{~K}, M_{\text {cont }}=80 M_{\odot}, N_{870}\left(\mathrm{H}_{2}\right)=1.08 \pm 0.05 \times 10^{22} \mathrm{~cm}^{-2}, N_{870} / N_{8}=3.0 \pm 2.0$, and $\left\langle n\left(\mathrm{H}_{2}\right)\right\rangle=0.4 \times 10^{4} \mathrm{~cm}^{-3}$.

Table 7. Reddened 2MASS point sources within the IRDC G304.74.

\begin{tabular}{|c|c|c|c|c|c|c|c|c|}
\hline 2MASS designation & $\begin{array}{c}J \\
{[\mathrm{mag}]}\end{array}$ & $\begin{array}{c}H \\
{[\mathrm{mag}]}\end{array}$ & $\begin{array}{c}K_{\mathrm{s}} \\
{[\mathrm{mag}]}\end{array}$ & $\begin{array}{l}J-K_{\mathrm{s}} \\
{[\mathrm{mag}]}\end{array}$ & $\begin{array}{l}E_{J-K}^{a} \\
{[\mathrm{mag}]}\end{array}$ & $\begin{array}{r}N_{2 \mathrm{MASS}}\left(\mathrm{H}_{2}\right)^{b} \\
{\left[10^{22} \mathrm{~cm}^{-2}\right]}\end{array}$ & $\begin{array}{c}N_{870}\left(\mathrm{H}_{2}\right) \\
{\left[10^{22} \mathrm{~cm}^{-2}\right]}\end{array}$ & $N_{870} / N_{2 \text { MASS }}$ \\
\hline $13064180-6128529$ & $14.15 \pm 0.04$ & $12.79 \pm 0.05$ & $12.04 \pm 0.04$ & $2.11 \pm 0.06$ & $1.0-1.5$ & $0.53-0.80$ & 2.3 & $2.9-4.3$ \\
\hline $13064266-6128213$ & $15.98 \pm 0.08$ & $14.42 \pm 0.04$ & $13.53 \pm 0.05$ & $2.44 \pm 0.10$ & $1.3-1.8$ & $0.71-0.98$ & 1.4 & $1.4-2$ \\
\hline $13070908-6124303$ & $14.26 \pm 0.02$ & $11.70 \pm 0.02$ & $10.39 \pm 0.02$ & $3.87 \pm 0.03$ & $2.7-3.2$ & $1.5-1.7$ & 0.62 & $\sim 0.4$ \\
\hline
\end{tabular}

Notes. ${ }^{(a)}$ Assuming $(J-K)_{0}=0.63-1.13$ corresponding to spectral classes K0 III-M3 III.

(b) Assuming $N\left(\mathrm{H}_{2}\right) / E_{J-K}=5.4 \times 10^{21} \mathrm{~cm}^{-2} \mathrm{mag}^{-1}$.

\subsection{Extinction estimates with $2 M A S S$}

We examined if extinctions estimated from the $J H K_{\mathrm{s}}$ photometry of 2MASS stars lying in the region can be used to calibrate the $\mathrm{H}_{2}$ column densities derived above ${ }^{7}$. A rarefaction of 2MASS point sources can be discerned in the cloud region and its immediate vicinity, and it is not possible to derive an extinction map of such detail that a comparison between the LABOCA map would be meaningful. Altogether 14 2MASS point sources with good photometric quality in all three bands lie within the LABOCA contour $0.1 \mathrm{Jy}$ beam $^{-1}$. Three of them show $J-H$ and $H-K_{\mathrm{s}}$ colours characteristic of giant stars reddened by substantial columns of interstellar dust. This judgement is based on their locations near the standard reddening line on the $J-H$ vs. $H-K_{\mathrm{s}}$ plot, and on the fact that their $J-K_{\mathrm{s}}$ excesses, $E_{J-K}$, are larger than 0.6 (see below). The three stars are the 2MASS PSC objects 13064180-6128529, 13064266-6128213, and 130709086124303 (see Table 7 and Fig. 6). The first two lie near the clump SMM 4 and are likely to represent background $\mathrm{K}$ or $\mathrm{M}$ giants. The third is found very close $\left(\sim 10^{\prime \prime}\right)$ to IRAS $13039-6108$ and is somewhat too bright for a class III giant in view of the distance and extinction. Because its NIR colours $(J-H=2.56 \pm 0.03$, $H-K_{\mathrm{s}}=1.31 \pm 0.03$ ) are consistent with an YSO (young stellar object) candidate with NIR excess (Matsuyanagi et al. 2006), the source is possibly a NIR counterpart of IRAS 13039-6108.

The $\mathrm{H}_{2}$ column density ranges given in Col. (7) of Table 7 are derived using intrinsic colours for K0 III-M3 III giants (Bessell \& Brett 1988), and the relationship $N\left(\mathrm{H}_{2}\right) / E_{J-K}=5.4 \times$ $10^{21} \mathrm{~cm}^{-2} \mathrm{mag}^{-1}$ (Bohlin et al. 1978; Mathis 1990; Harjunpää \& Mattila 1996). The colour excesses, $E_{J-K}$, measure the total

7 The 2MASS All-Sky Point Source Catalog (PSC) used here have been made available at http://irsa.ipac. caltech.edu/

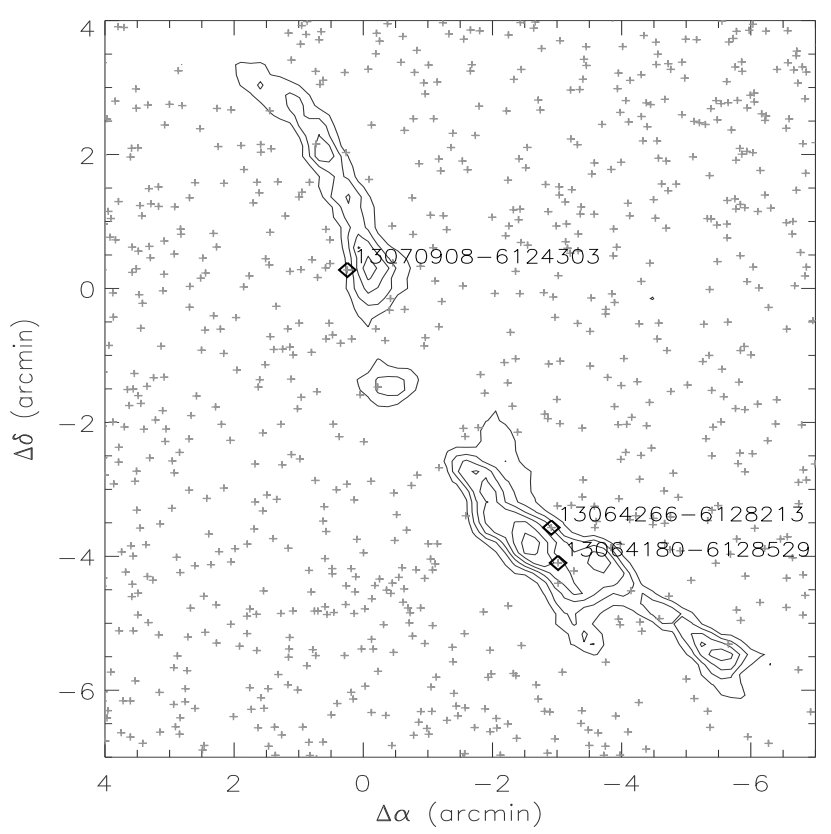

Fig. 6. LABOCA dust continuum map (contours) with 2MASS point sources (plus signs). The heavily reddened 2MASS stars within the cloud boundaries are marked with diamonds. The contour levels go from 0.1 to 0.6 by $0.1 \mathrm{Jy}$ beam $^{-1}$.

amount of dust in front of the stars, so they are affected by dust in the foreground, and possibly also in the background of the cloud. Therefore, the $N\left(\mathrm{H}_{2}\right)$ values are upper limits for the contribution of the cloud itself. A nearby line of sight $(l=304.75, b=1.25)$ has been included in the investigation of 3-D distribution of the 
extinction by Marshall et al. (2006). Their results suggest a foreground extinction of $A_{K} \sim 0.4\left(E_{J-K} \sim 0.6\right.$; Marshall et al. 2006; Mathis 1990) up to the cloud's distance $2.4 \mathrm{kpc}$.

The LABOCA $870 \mu \mathrm{m}$ intensities in the directions of 13064180-6128529, 13064266-6128213, and 130709086124303 are $0.49,0.31$, and $0.13 \pm 0.03 \mathrm{Jy}$ beam $^{-1}$, respectively. The conversion to the $\mathrm{H}_{2}$ column densities using Eq. (8) with $T_{\mathrm{d}}=15 \mathrm{~K}$ gives $2.3 \times 10^{22}, 1.4 \times 10^{22}$, and $0.62 \times 10^{22} \mathrm{~cm}^{-2}$. The first two values are not consistent with the upper limits derived from $E_{J-K}$ (see Table 7, Col. (9)). In the case of 130641806128529 , the $\mathrm{H}_{2}$ column density derived from LABOCA $870 \mu \mathrm{m}$ data can be brought down to conform with 2MASS estimate by increasing the dust temperature to $T_{\mathrm{d}}=30 \mathrm{~K}$, whereas for the location of 13064266-6128213 an increase to $T_{\mathrm{d}}=20 \mathrm{~K}$ would be sufficient. The latter value is in good agreement with the bolometric temperature of IRAS $13037-6112\left(T_{\text {cold }} \simeq 22 \mathrm{~K}\right.$, see Table 5, Col. (4)) which lies (in the plane of the sky) quite close to $13064266-6128213$. The $8 \mu \mathrm{m}$ intensities in the directions of $13064180-6128529$ and $13064266-6128213$ are $1.03 \times 10^{-6}$ and $1.22 \times 10^{-6} \mathrm{~W} \mathrm{~m}^{-2} \mathrm{sr}^{-1}$, respectively. These correspond to $8 \mu \mathrm{m}$ optical thicknesses of $0.41 \pm 0.19$ and 0.02 (here the associated error is larger than the value), respectively (see Eq. (2)). Using Eq. (9), the corresponding $\mathrm{H}_{2}$ column densities become $0.59 \pm 0.27 \times 10^{22}$ and $0.03 \times 10^{22} \mathrm{~cm}^{-2}$, respectively. The first value is consistent with the 2 MASS estimate, whereas the latter value is much lower.

The above results clearly show that it is important to know the dust temperature in order to accurately determine the $\mathrm{H}_{2}$ column density from the submm dust continuum emission (Eq. (8)). On the other hand, the MIR absorption and 2MASS extinction methods require several uncertain assumptions, such as the dust model and the corresponding dust extinction cross-section, contribution of the foreground emission, and the relation between $N\left(\mathrm{H}_{2}\right)$ and $E_{J-K}$. Moreover, as the 2MASS extinction could be estimated only along a few lines of sight, the present statistics is very poor. Thus, the data presented here do not offer a firm conclusion about the most reliable method to determine the $\mathrm{H}_{2}$ column densities. We note that the empirical relationship between $N_{\mathrm{H}}$ and $A_{J}$ derived by Vuong et al. (2003) in $\rho$ Oph, used recently by Marshall et al. (2009), implies $N\left(\mathrm{H}_{2}\right) / E_{J-K} \simeq$ $4.7 \times 10^{21} \mathrm{~cm}^{-2} \mathrm{mag}^{-1}$. This would make the $\mathrm{H}_{2}$ column densities about $13 \%$ smaller than those estimated above.

\section{Discussion}

\subsection{Estimating the background and foreground MIR emission}

The average MIR emission around the cloud, $I_{\mathrm{MIR}}=1.23 \pm$ $0.02 \times 10^{-6} \mathrm{~W} \mathrm{~m}^{-2} \mathrm{sr}^{-1}$, was estimated in Sect. 4 by using median filtering. Vasyunina et al. (2009) estimated $I_{\text {MIR }}$ in the vicinity of several IRDCs directly from the $8 \mu \mathrm{m}$ images. They pointed out that it is difficult to control the process of median filtering if large $\left(\gtrsim 10^{\prime}\right)$ filters are needed. On the other hand, if the filter is too small (comparable to the cloud size), the value of $I_{\text {MIR }}$ will be underestimated because it will be affected by the cloud itself (Butler \& Tan 2009). Because the largest diameter of G304.74 is $\sim 13^{\prime}$, we tried the direct method by choosing manually MIR emission patches (free of strong MIR emission sources) in the close vicinity of the IRDC. We used three different patches with angular sizes of 6 .' $7 \times 3$.' 3 , 4 .' $8 \times 2$ '. 3 , and 5. . $7 \times 1$ ". 8 . The mean and standard deviation of the $8 \mu \mathrm{m}$ intensity within these regions were $1.17 \pm 0.18 \times$ $10^{-6}, 1.21 \pm 0.18 \times 10^{-6}$, and $1.25 \pm 0.21 \times 10^{-6} \mathrm{~W} \mathrm{~m}^{-2} \mathrm{sr}^{-1}$, respectively. The mean and standard deviation of these three are $1.21 \pm 0.11 \times 10^{-6} \mathrm{~W} \mathrm{~m}^{-2} \mathrm{sr}^{-1}$. This result is in excellent agreement with the value obtained from median filtering.

Based on the observed anti-correlation between the $850 \mu \mathrm{m}$ and $8 \mu \mathrm{m}$ intensities (cf. Fig. 4), Johnstone et al. (2003) and Ormel et al. (2005) found that $I_{\text {fore }} \simeq I_{\mathrm{bg}}$ in the case of IRDC G11.11-0.12 and the W51 IRDC, respectively. Peretto \& Fuller (2009) constrained $I_{\text {fore }}$ by the requirement that both MIR absorption and $1.2 \mathrm{~mm}$ emission should give the same $N\left(\mathrm{H}_{2}\right)$. In this method, it is assumed that the true $8 \mu \mathrm{m}$ opacity can be calculated from the millimetre emission (see Eq. (3) of Peretto \& Fuller 2009). Then, the value of $I_{\text {fore }}$ can be calculated in terms of $I_{\text {MIR }}$ (see our Eq. (2)). Peretto \& Fuller (2009) also showed that on the average the background emission is approximately equal to the foreground emission (their Eq. (5)). The drawback in this approach is the uncertainty in the ratio of the mass absorption coefficients at MIR and (sub)mm wavelengths $\left(R_{\kappa}\right.$ in Eq. (3) of Peretto \& Fuller 2009). In the present study, we estimated the contributions of background and foreground emission using the foreground intensity ratio (Sect. 4.2). We also ended up with the result that $I_{\text {fore }} \simeq I_{\mathrm{bg}}$. Butler \& Tan (2009) and RBG09 estimated that $I_{\text {fore }} \simeq(0.1-0.5) \times I_{\text {bg }}\left(f_{\text {fore}}\right.$-method $)$ and $I_{\text {fore }}=(2-5) \times I_{\text {bg }}(850-8 \mu \mathrm{m}$ anti-correlation $)$ for their samples of IRDCs, respectively.

\subsection{Comparison of $\mathrm{H}_{2}$ column densities determined from dust continuum emission and extinction data}

The $\mathrm{H}_{2}$ column densities derived from submm emission and MIR absorption are mostly in good agreement (within a factor of $\sim 2$; Table 6, Cols. (4) and (5)). This suggests that the dust parameters used in Eqs. (8) and (9) are reasonable. The dust temperature, $T_{\mathrm{d}}$, is likely to show spatial variations, and this causes uncertainties to column density estimates based on submm emission (Eq. (8)), but does not affect the $8 \mu \mathrm{m}$ absorption method (Eq. (9)). The greatest differences between the two $N\left(\mathrm{H}_{2}\right)$ values are found toward positions with the highest $N_{870}\left(\mathrm{H}_{2}\right)$ values. These are SMM 1, 3, and 4, where the $N_{870}\left(\mathrm{H}_{2}\right) / N_{8}\left(\mathrm{H}_{2}\right)$ ratios are $2.8 \pm 1.1,16 \pm 0.9$, and $8.2 \pm 5.5$, respectively (Col. (6) of Table 6). Some of these discrepancies could (in part) be remedied, e.g., by increasing the dust temperature (cf. footnote in Table 6). It seems possible that the dust temperature is higher than $15 \mathrm{~K}$ in the four clumps (SMM 1-4) near southwestern tip of the cloud. On the other hand, Vasyunina et al. (2009) showed that the extinction method becomes unreliable at very high column densities, but this should happen only at $N\left(\mathrm{H}_{2}\right) \gtrsim 10^{23} \mathrm{~cm}^{-2}$.

However, there are also uncertainties in deriving $N_{8}\left(\mathrm{H}_{2}\right)$, in particular related to the contamination by the foreground emission (here done by using the foreground intensity ratio, see Sect. 4), and the dust extinction cross-section (reliable only within a factor of $\sim 2$, e.g., Ragan et al. 2006). Also, the value of $\kappa_{\lambda}$, needed in the calculation of $N_{870}\left(\mathrm{H}_{2}\right)$ (Eq. (8)) has an uncertainty similar to that of $\sigma_{\lambda}$. The dust-to-gas mass ratio could also differ from the adopted value $1 / 100$ as mentioned in Sect. 5.1. Thus, the direct comparison of $N\left(\mathrm{H}_{2}\right)$ derived from dust continuum and extinction data should be taken with caution. We note that Parsons et al. (2009) found for their sample of IRDCs that $N_{8}\left(\mathrm{H}_{2}\right)(\mathrm{MSX})$ and $N_{850}\left(\mathrm{H}_{2}\right)$ (SCUBA) agree within an order of magnitude. The moderate correspondence may be partly explained by the omission of the foreground emission. 


\subsection{Nature of submm clumps}

The MSX $8 \mu \mathrm{m}$ emission associated with molecular clumps suggests the presence of protostars. By combining the submm LABOCA and MIR MSX data one can distinguish between candidate starless and star-forming clumps. Of the twelve clumps in G304.74, four are likely to be associated with newly born stars. In addition to the three IRAS (and MSX) sources, the clump SMM 6 is associated with two MSX $8 \mu \mathrm{m}$ point sources. The remaining eight MIR dark clumps are either starless or contain low-luminosity (low-mass) YSOs falling under the detection limit of the MSX (cf. Parsons et al. 2009). For instance, the Spitzer/GLIMPSE survey with better sensitivity and resolution compared to that of MSX, revealed that some IRDC clumps previously thought to be starless do contain MIR sources (e.g., Chambers et al. 2009). Moreover, Chambers et al. (2009) detected $\mathrm{H}_{2} \mathrm{O}$ masers towards some MIR dark clumps, which is a clear indication of star formation activity in them. On the other hand, starless clumps may be either prestellar or just unbound structures that will eventually disperse.

The extremely short lifetime of starless IRDC clumps $\left(10^{3}-10^{4} \mathrm{yr}\right.$, i.e., $\sim$ half of the time spent in the protostellar phase) derived by Parsons et al. (2009) does not support the idea that all of the eight dark clumps in G304.74 could be starless. If gravitationally bound, these clumps with masses in the range $\sim 40-200 M_{\odot}$ (Table 6, Col. (3)) are capable of forming high-mass stars (Thompson et al. 2006), and some of them could represent/contain HMSCs (cf. Chambers et al. 2009). The peak column densities of the clumps do not reach the minimum column density threshold of $1 \mathrm{~g} \mathrm{~cm}^{-2}$, i.e., $N\left(\mathrm{H}_{2}\right) \sim 2.2 \times 10^{23} \mathrm{~cm}^{-2}$, proposed by Krumholz \& McKee (2008) for the formation of high-mass stars. However, it is possible that the clumps host cores where such a high $N\left(\mathrm{H}_{2}\right)$ values can be reached (e.g., Hennemann et al. 2009).

The presence of high-luminosity $\left(\sim 1.5-2 \times 10^{3} L_{\odot}\right)$ IRAS sources indicates that intermediate to high-mass star formation is going on in other parts of the cloud. In fact these luminosities suggest intermediate-mass stars as high-mass protostellar objects (HMPOs) are often found in the range $\sim 10^{3.5}-10^{5.5} L_{\odot}$ (e.g., Sridharan et al. 2002; Fazal et al. 2008; Grave \& Kumar 2009). On the other hand, the envelope masses of these sources ( 60-90 $M_{\odot}$, see Col. (2) of Table 5) are sufficiently large for high-mass star formation (Beuther \& Steinacker 2007). For comparison, majority of the low-mass protostars in nearby $(d \lesssim$ 250 pc) molecular clouds have luminosites $L_{\text {bol }} \leq$ a few $\times L_{\odot}$, the highest observed values being $\sim 70 L_{\odot}$ in only a few cases (e.g., Evans et al. 2009). IRAS 13037-6112 and IRAS 130396108 have similar SED parameters (see Fig. 5 and Table 5), suggesting that they probably represent the same evolutionary stage. Based on its IRAS [25-12] and [60-12] colours (0.61 and 1.55 , respectively), IRAS $13037-6112$ belongs to the so-called High sources, and could potentially be associated with ultracompact (UC) HII region (e.g., Wood \& Churchwell 1989; Palla et al. 1991; Molinari et al. 1996). However, the $M / L$ ratio of IRAS 13037-6112(0.04) is more typical of sources younger than UC HII regions (Sridharan et al. 2002). IRAS 13039-6108 has a [25-12] colour index of 0.27 , settling it to the so-called Low sources. Moreover, Fontani et al. (2005) found that the $\mathrm{C}^{17} \mathrm{O}$ and CS linewidths in IRAS 13039-6108 are significantly smaller than those typically observed in massive clumps associated with UC HII regions (e.g., Cesaroni et al. 1991; Hofner et al. 2000). This further supports the idea that both IRAS 13037-6112 and IRAS 13039-6108 represent the same evolutionary stage (earlier than UC HII). Using the assumption of an isothermal sphere

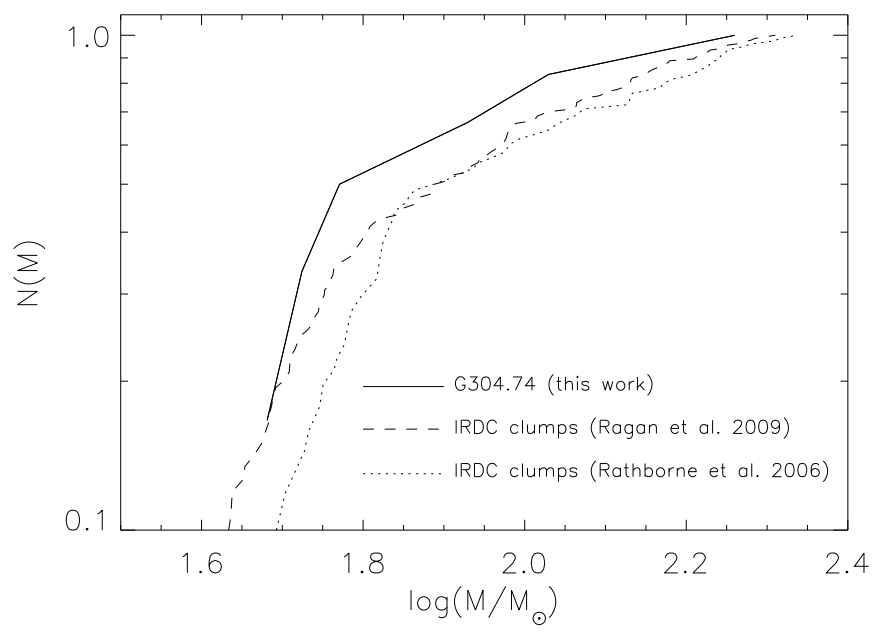

Fig. 7. Normalised cumulative mass functions, $\mathcal{N}(M)$, for the MIR dark clumps in the IRDC G304.74 (solid line) and in several other IRDCs studied by RJS06 (dotted line), and by RBG09 (dashed line). Clump masses from RJS06 and RBG09 were scaled down to match our assumptions about $T_{\mathrm{d}}$ and $\kappa_{\lambda}$ (see main text)

with $T_{\text {kin }}=22 \mathrm{~K}$ and a density profile of the form $n(r) \propto r^{-1.6}$, which is typical of high-mass star-forming clumps (e.g., Beuther et al. 2002a), the $\mathrm{C}^{17} \mathrm{O}(2-1)$ linewidth of $0.93 \mathrm{~km} \mathrm{~s}^{-1}$ observed by Fontani et al. (2005) implies a virial mass of $\sim 99 M_{\odot}$ for IRAS $13039-6108\left(M_{\text {cont }} / M_{\text {vir }} \simeq 0.8, M_{\mathrm{SED}} / M_{\text {vir }} \simeq 0.9\right.$, i.e., the virial parameter defined by Bertoldi \& McKee (1992) is $\alpha_{\mathrm{vir}}=$ $M_{\text {vir }} / M \simeq 1.1-1.3$ ) (see, e.g., Eqs. (1) and (2) of Chen et al. 2008). Thus the clump is near virial equilibrium. IRAS 130426105 in the northern part of the cloud is probably in an earlier stage of evolution than the other two IRAS sources because it is not as bright at FIR wavelengths (see Table 3).

\subsection{Clump mass distribution}

The mass distribution of clumps/cores is important parameter concerning the cloud fragmentation mechanism. Our sample of clumps is, however, so small (12 in total, 8 MIR dark) that it is not reasonable to study their mass distribution directly. Therefore, we only compared it with the mass distributions derived for other, larger IRDC clump samples, using the results of Sridharan et al. (2005), RJS06, Vasyunina et al. (2009), and RBG09.

Figure 7 presents the observed cumulative mass functions, which include clumps of mass less than $M$, i.e., $\mathcal{N}(M)=N(m<$ $M) / N_{\text {tot }}$, for G304.74 and for a sample of cold clumps from RJS06 and RBG09. We note that the IRDC clump mass functions in RJS06 and in our work are constructed by removing the MIR bright clumps from the samples (in the case of G304.74, this means the three IRAS sources and SMM 6). From the sample of Sridharan et al. (2005), we excluded the high temperature clump No. 9, because its temperature $(32.7 \mathrm{~K})$ was much higher than the rest of the sample. From the the sample of RBG09, we removed the clumps associated with YSOs, and the clumps possibly contaminated by foreground (or background) stars. Thus, the mass functions include only those clumps that initially have all their mass available for star formation.

The previous studies taken into this comparison used slightly different assumptions about the dust temperature and opacity. We scaled all the clump masses included in the cumulative mass functions to correspond to a uniform dust temperature of 
Table 8. K-S test results between different IRDC clump mass distributions.

\begin{tabular}{cccc}
\hline \hline & $\begin{array}{c}\text { Number of } \\
\text { cold clumps }\end{array}$ & $D_{\max }$ & Prob. \\
\hline Study & 345 & 0.153 & 0.998 \\
Ragan et al. (2009, RBG09) $^{a}$ & 140 & 0.272 & 0.739 \\
Rathborne et al. (2006, RJS06) $^{a}$ & 28 & 0.359 & 0.534 \\
Sridharan et al. (2005) & 17 & 0.449 & 0.408 \\
Vasyunina et al. (2009) & &
\end{tabular}

Notes. ${ }^{(a)}$ The probability that the RBG09 and RJS06 clump mass samples represent the subsamples of the same parent distribution is $100 \%$ $\left(D_{\max }=0.001\right)$.

$T_{\mathrm{d}}=15 \mathrm{~K}$, and an opacity that is consistent with our $\kappa_{870}=$ $0.17 \mathrm{~m}^{2} \mathrm{~kg}^{-1}$. RBG09 derived clump masses from the total hydrogen column densities, $N_{\mathrm{H}}$, as estimated from the $8 \mu \mathrm{m}$ optical thicknesses, $\tau_{8}$. For the dust extinction cross-section per $\mathrm{H}$ nucleus at $8 \mu \mathrm{m}, \sigma_{8}$, they used the value $2.3 \times 10^{-23} \mathrm{~cm}^{2}$, based on the Weingartner \& Draine (2001) dust model. According to the model we have used (Ossenkopf \& Henning 1994), the corresponding number is $3.5 \times 10^{-23} \mathrm{~cm}^{2}$ per $\mathrm{H}$ nucleus (see Sect. 5.3). In their mass formula, RBG09 used a factor of 1.16 as the ratio of the total gas mass (including $\mathrm{He}$ ) and the hydrogen mass. In our calculations this ratio has been 1.4 (Sect. 5.3). These differences have been accounted for by scaling the masses from RBG09 by 0.8 in the comparison with our results.

To determine whether our clump masses and those from other studies are derived from the same clump mass distribution, we carried out the two-sample Kolmogorov-Smirnov (K-S) test. For this test, the mass scales were matched, i.e., the comparison was done within the range of common mass interval. The K-S test results are shown in Table 8 . The columns of this table are: (1) the survey used in the comparison; (2) number of clumps included; (3) the maximum vertical difference between the cumulative mass distributions $\left(D_{\max }\right)$; $(4)$ the probability for the null hypothesis that the two functions are drawn form the same parent distribution (the significance level of the K-S statistic).

The highest number statistic is provided by the study of RBG09, and when compared with this study, the K-S test yields a probability of $99.8 \%$ that the clump mass distributions in G304.74 and the other IRDCs are drawn from the same parent distribution. This probability drops significantly when smaller samples are used in the test (Table 8, Col. (4)). Moreover, according to the K-S test, there is a $100 \%$ probability $\left(D_{\max }=\right.$ 0.001) that the RBG09 and RJS06 clump masses represent the subsamples of the same underlying parent distribution. For this test, clump masses from RBG09 were also multiplied by 0.8 to compare with the RJS06 masses which were based on the value $\kappa_{1.2 \mathrm{~mm}}=0.1 \mathrm{~m}^{2} \mathrm{~kg}^{-1}$.

For the clump masses between $\sim 30$ and $3000 M_{\odot}$, RBG09 derived an IRDC clump mass spectrum with a slope of $\alpha=$ $1.76 \pm 0.05$, which is consistent with the mass functions derived for high-mass star-forming regions, and also resembles the mass function of Galactic stellar clusters (see RBG09 and references therein). Note, however, that RBG09 merged all their clumps into a single mass function, whereas we have used only starless clumps in deriving the cumulative mass functions. RJS06 found a Salpeter-like ( $\alpha=2.35$; Salpeter 1955) mass function for their cold IRDC clumps $\left(\alpha=2.1 \pm 0.4\right.$ at $\left.M \gtrsim 100 M_{\odot}\right)$. The IRDC clump mass spectra derived by RJS06 and RBG09 are comparable to those predicted by turbulent fragmentation models (see Sect. 6.7 and references therein).

\subsection{Clump spatial distribution}

In addition to the clump mass distribution, it is also useful to determine how clumps are spatially distributed within the cloud in order to unveil the presence of a possible preferred length-scale of fragmentation (e.g., Muñoz et al. 2007). For this purpose, we determined the clump-separation distributions and the number distributions of the projected separation distance between nearest neighbours ${ }^{8}$ in G304.74, and in nine other IRDCs for comparison. We chose those IRDCs from the sample of RJS06 which contain the largest number of clumps, i.e., MSXDC G023.60+00.00, G024.33+00.11, G028.37+00.07, G028.5300.25, G031.97+00.07, G033.69-00.01, G034.43+00.24, and G035.39-00.33. Moreover, we determined the spatial distribution of YSOs in the IRDC MSXDC G048.65-00.29 studied by van der Wiel \& Shipman (2008). For these analyses, we selected only those sources that are clearly associated (in the plane of the sky) with their parental dark cloud (e.g., clumps that lie within the dark filaments). Thus, we excluded the millimetre clumps MM 1, 3, and 5 from G023.60+00.00, MM 2, 5, and 7 from G024.33+00.11, MM 3, 5, 7, 8, 12, 13, 18 from G028.37+00.07, MM 1 and 2 from G035.39-00.33, and YSOs S4, 10, 17, 18, 19, and 20 from G048.65-00.29. In addition to the observed spatial distributions, we also determined the distributions expected from random positions of the same number of objects as the observed samples have. The areas over which the objects were randomly distributed were chosen so that they approximate the observed dark cloud areas; the IRDC areas were estimated by rectangles which just cover the observed dark clouds. When needed, these rectangles were rotated with respect to the $(\alpha, \delta)$-coordinate system. The random distributions were generated a hundred times per cloud and the resulting averaged histograms were used in comparisons with observed spatial distributions.

Figure 8 (top) shows the observed clump-separation distribution in G304.74, and the distribution expected for the same number of randomly positioned clumps over minimum rectangular area which encloses the dark cloud ( 29.8 $\left.\operatorname{arcmin}^{2}\right)$. The mean and its standard deviation, and median of the clump separations in $\mathrm{G} 304.74$ are $\log \left(\langle r\rangle_{\text {obs }} / \mathrm{AU}\right)=5.690 \pm 0.041\left(4.90_{-0.44}^{+0.48} \times\right.$ $\left.10^{5} \mathrm{AU}\right)$ and $\log \left(\tilde{r}_{\text {obs }} / \mathrm{AU}\right)=5.759\left(5.74 \times 10^{5} \mathrm{AU}\right)$, respectively. These values are similar to those of randomly positioned clumps, for which the mean and median are $\log \left(\langle r\rangle_{\mathrm{ran}} / \mathrm{AU}\right)=$ $5.674 \pm 0.061$ and $\log \left(\tilde{r}_{\text {ran }} / \mathrm{AU}\right)=5.738 \pm 0.084($ see Table 9$)$. The latter two values and their \pm -errors quoted represent the average values and their standard deviations derived from the 100 generated random distributions. According to the K-S test, the probability that the observed distribution and the generated random distribution represent the same underlying distribution is $100 \%$. Statistics of the clump-separation distributions in other IRDCs studied by RJS06 and van der Wiel \& Shipman (2008) are listed in Table 9. The columns of this table are the following: (1) IRDC name; (2) number of clumps used in the analysis (see above); (3) distance; (4) area used to create the random distributions (see above); (5) and (6) mean and median of the observed clump-separation distribution $\left(\langle r\rangle_{\mathrm{obs}}\right.$ and $\left.\tilde{r}_{\mathrm{obs}}\right)$; (7) and (8) mean and median of the corresponding random distribution $\left(\langle r\rangle_{\text {ran }}\right.$ and $\left.\tilde{r}_{\text {ran }}\right) ;(9)$ and (10) ratios between the observed and random mean and median separations (quoted errors are propagated from the standard deviations of $\langle r\rangle$ and $\tilde{r}$ ); (11) probability given by the K-S test that the observed and random

\footnotetext{
8 If the number of clumps in the cloud is $N_{\mathrm{cl}}$, then the number of clump-separations is $N_{\mathrm{cl}}\left(N_{\mathrm{cl}}-1\right) / 2$, whereas the number of distances between an individual clump and its nearest neighbour is equal to $N_{\mathrm{cl}}$.
} 

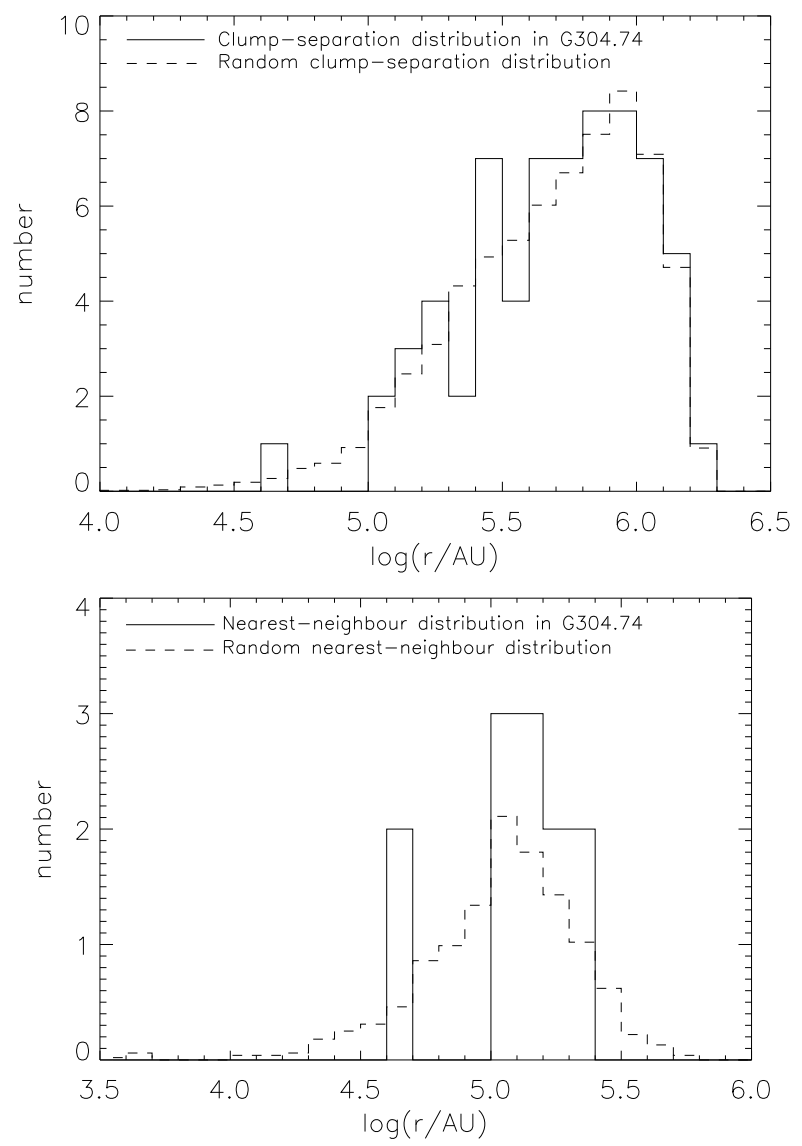

Fig. 8. Top: observed clump-separation distribution (solid line) compared with the expected distribution for random distribution of the same number of clumps as the observed sample over rectangular area which approximate the area of the IRDC (dashed line). Bottom: observed nearest-neighbour distribution (solid line) compared with the expected distribution for random distribution (dashed line).

distributions are drawn from the same underlying distribution. The observed clump separations are mostly similar to those expected from random distributions. This is evident from the ratios $\langle r\rangle_{\text {obs }} /\langle r\rangle_{\text {ran }}$ and $\tilde{r}_{\text {obs }} / \tilde{r}_{\text {ran }}$ which are close to unity, and from the K-S probabilities which are high $(\sim 71-100 \%)$ except for three cases (G035.39, G028.37, G024.33; see Cols. (9)-(11) of Table 9).

Figure 8 (bottom) compares the observed nearest-neighbour distribution in G304.74 with the distribution for randomly positioned clumps. The mean and median of the nearest-neighbour distribution in G304.74 are $\log \left(\langle r\rangle_{\text {obs }} / \mathrm{AU}\right)=5.083 \pm 0.058$ $\left(1.21_{-0.15}^{+0.17} \times 10^{5} \mathrm{AU}\right)$ and $\log \left(\tilde{r}_{\text {obs }} / \mathrm{AU}\right)=5.136\left(1.37 \times 10^{5} \mathrm{AU}\right)$, respectively. Again, these values are comparable to those of randomly positioned clumps, for which the mean and median are $\log \left(\langle r\rangle_{\mathrm{ran}} / \mathrm{AU}\right)=5.030 \pm 0.111$ and $\log \left(\tilde{r}_{\mathrm{ran}} / \mathrm{AU}\right)=5.047 \pm$ 0.110 , respectively (see Table 10 ). According to the K-S test, there is about $90 \%$ probability that the observed and random nearest-neighbour distributions are samples of the same underlying distribution. We note that the minimum observable separation corresponds to the beam size, i.e, $18^{\prime \prime}$. 6 or $\sim 4.46 \times$ $10^{4} \mathrm{AU}(\log (r / \mathrm{AU})=4.649)$ at $2.4 \mathrm{kpc}$. Statistics of the nearestneighbour distributions in other IRDCs are given in Table 10. Table 10 have the same meaning as in Table 9, except now for nearest neighbour separations. The observed nearest-neighbour distances are similar to those expected from random distributions. This is evident from the ratios $\langle r\rangle_{\mathrm{obs}} /\langle r\rangle_{\mathrm{ran}}$ and $\tilde{r}_{\mathrm{obs}} / \tilde{r}_{\mathrm{ran}}$ which are (within the erros) about 1 , and by the high K-S probabilities ( 59-100\%) in every other case except G031.97, where this probability is still $\sim 37 \%$ (see Cols. (6)-(8) of Table 10).

In summary, the average projected separations between clumps in the studied IRDCs range from about $2.6 \times 10^{5} \mathrm{AU}$ to $1.2 \times 10^{6} \mathrm{AU}$ (i.e., the minimum and maximum lie within a factor of five), and the average projected distances between the nearest neighbours range from $6.0 \times 10^{4} \mathrm{AU}$ to $3.5 \times 10^{5} \mathrm{AU}$ (i.e., the changes are within a factor of six). For most clouds, the distributions of projected separations and distances between the nearest neighbours can be mimicked by clumps placed randomly into the same projected area as occupied by the cloud. Assuming that the vectors connecting clump pairs are randomly oriented, the average projection factor is $\left\langle\sin \theta_{i j}\right\rangle=\pi / 4$, where $\theta_{i j}$ is the angle between the line of sight and the vector pointing from clump $i$ to clump $j$. Correcting for this projection effect, the grand averages of the separations and distances between the nearest neighbours are about $6.5 \times 10^{5} \mathrm{AU}(3.1 \mathrm{pc})$ and $2.2 \times 10^{5} \mathrm{AU}(1.1 \mathrm{pc})$, respectively.

\subsection{Fragmentation of IRDCs}

One plausible scenario for the origin of filamentary clouds is that they are formed in shocks occurring in converging flows driven by large-scale turbulence (e.g., Klessen et al. 2000; Padoan et al. 2001). The chaotic process can give rise to randomly positioned density peaks within filaments, and these can become centres of gravitational collapse. On the other hand, supposing that compression leads to an equilibrium structure, a filament can fragment through the Jeans instability.

The critical wavelength, $\lambda_{c}$, of perturbations leading to gravitational instability depends on both the gas kinetic tempeture, $T_{\text {kin }}$, and the density, $\rho: \lambda_{\mathrm{c}} \sim c_{\mathrm{s}} / \sqrt{G \rho}$, or, in terms of the surface density, $\Sigma: \lambda_{\mathrm{c}} \sim c_{\mathrm{s}}^{2} / G \Sigma$, where $c_{\mathrm{s}}$ is the sound speed, and $G$ is the gravitational constant (e.g., Larson 1985; Hartmann 2002).

The determination of the "Jeans length" is not quite straightforward in a study based on dust emission because the cloud mass and therefore also the average density and surface density depend on the assumed dust temperature, $T_{\mathrm{d}}$. Furthermore, in the case of G304.74, there is no independent estimate of $T_{\text {kin }}$, but it is assumed to be equal to $T_{\mathrm{d}}$.

The assumption $T_{\mathrm{d}}=15 \mathrm{~K}$ yields a total mass of $\sim 1000 M_{\odot}$ and an average surface density of $0.05 \mathrm{~g} \mathrm{~cm}^{-2}$ for $\mathrm{G} 304.74$ (within the LABOCA contour $0.1 \mathrm{Jy}$ beam $^{-1}$ ). In these circumstances, the critical wavelength in an isothermal equilibrium filament is $\lambda_{\mathrm{c}}=0.19 \mathrm{pc}$ or $40000 \mathrm{AU}$ (Hartmann 2002; Larson $1985)$, and the corresponding mass is $M_{\mathrm{c}} \sim 5 M_{\odot}$. The comparison between dust emission at $870 \mu \mathrm{m}, 8 \mu \mathrm{m}$ absorption, and the visual extinction from 2 MASS suggests an elevated temperature in the southern part of the cloud (Table 4, Col. (4)). By assuming $T_{\mathrm{d}}=30 \mathrm{~K}$, one would obtain a total cloud mass of $\sim 400 M_{\odot}$, and an average surface density of $\Sigma=0.02 \mathrm{~g} \mathrm{~cm}^{-2}$. These values of temperature and surface density imply $\lambda_{\mathrm{c}}=1.1 \mathrm{pc}$ or $2.2 \times 10^{5} \mathrm{AU}$, and $M_{\mathrm{c}} \sim 50 M_{\odot}$. The critical wavelength, $\lambda_{\mathrm{c}}$, is not expected to determine a uniform length scale of fragmentation. According to the analysis of Stodólkiewicz (1963; see also Curry 2000, and references therein), the fastest growing perturbations have a length scale of roughly twice $\lambda_{\mathrm{c}}$. Nevertheless, the fragmentation of a homogenous cloud is likely to result in a preferred length scale and a quasi-periodic structure, as opposed to a random distribution of clumps.

The projected distances between the nearest neighbours in G304.74 lie in the range $4 \times 10^{4}-2.4 \times 10^{5} \mathrm{AU}$. They are comparable to the characterics length scales indicated above. However, 
Table 9. Statistics of the clump-separation distributions in IRDCs.

\begin{tabular}{|c|c|c|c|c|c|c|c|c|c|c|}
\hline \multirow[b]{2}{*}{ Name MSXDC } & \multirow[b]{2}{*}{$N_{\mathrm{cl}}$} & \multirow[b]{2}{*}{$\begin{array}{c}d \\
{[\mathrm{kpc}]}\end{array}$} & \multirow[b]{2}{*}{$\begin{array}{c}\text { Area } \\
{\left[\square^{\prime}\right]}\end{array}$} & \multicolumn{2}{|c|}{ Observed distribution } & \multicolumn{2}{|c|}{ Random distribution } & \multirow[b]{2}{*}{$\langle r\rangle_{\mathrm{obs}} /\langle r\rangle_{\mathrm{ran}}$} & \multirow[b]{2}{*}{$\tilde{r}_{\text {obs }} / \tilde{r}_{\text {ran }}$} & \multirow[b]{2}{*}{ Prob. } \\
\hline & & & & $\begin{array}{c}\langle r\rangle_{\text {obs }} \\
{[\log \mathrm{AU}]}\end{array}$ & $\begin{array}{c}\tilde{r}_{\mathrm{obs}} \\
{[\log \mathrm{AU}]}\end{array}$ & $\begin{array}{c}\langle r\rangle_{\mathrm{ran}} \\
{[\log \mathrm{AU}]}\end{array}$ & $\begin{array}{c}\tilde{r}_{\text {ran }} \\
{[\log \mathrm{AU}]}\end{array}$ & & & \\
\hline G304.74+01.32 & 12 & 2.4 & 29.8 & $5.690 \pm 0.041$ & 5.759 & $5.674 \pm 0.061$ & $5.738 \pm 0.084$ & $1.04 \pm 0.18$ & $1.05 \pm 0.20$ & 1.00 \\
\hline G048.65-00.29 & $14^{a}$ & 2.5 & 13.5 & $5.482 \pm 0.035$ & 5.524 & $5.449 \pm 0.059$ & $5.497 \pm 0.071$ & 17 & 1.06 & 0.709 \\
\hline G035.39-00.33 & 7 & 2.9 & 17.2 & $5.422 \pm 0.058$ & 5.493 & $5.583 \pm 0.095$ & $5.630 \pm 0.117$ & $0.69 \pm 0.18$ & $0.73 \pm 0.20$ & 0.156 \\
\hline $\mathrm{G} 034.43+00.24$ & 9 & 3.7 & 12.6 & $5.608 \pm 0.051$ & 5.672 & $5.618 \pm 0.075$ & $5.670 \pm 0.096$ & $0.98 \pm 0.21$ & $1.00 \pm 0.22$ & 1.000 \\
\hline G033.69-00.01 & 11 & 7.1 & 28.7 & $6.089 \pm 0.044$ & 6.152 & $6.076 \pm 0.062$ & $6.123 \pm 0.083$ & $1.03 \pm 0.18$ & $1.05 \pm 0.20$ & 0.982 \\
\hline $\mathrm{G} 031.97+00.07$ & 9 & 6.9 & 18.1 & $5.861 \pm 0.051$ & 5.870 & $5.943 \pm 0.073$ & $5.995 \pm 0.088$ & $0.83 \pm 0.17$ & $0.75 \pm 0.15$ & 0.738 \\
\hline G028.53-00.25 & 10 & 5.7 & 17.6 & $5.733 \pm 0.042$ & 5.797 & $5.822 \pm 0.060$ & $5.879 \pm 0.070$ & $0.81 \pm 0.14$ & $0.83 \pm 0.13$ & 0.753 \\
\hline $\mathrm{G} 028.37+00.07$ & 11 & 5.0 & 51.5 & $5.903 \pm 0.033$ & 5.934 & $5.977 \pm 0.055$ & $6.044 \pm 0.065$ & $0.84 \pm 0.13$ & $0.78 \pm 0.12$ & 0.324 \\
\hline $\mathrm{G} 024.33+00.11$ & 8 & 3.8 & 27.5 & $5.772 \pm 0.061$ & 5.926 & $5.727 \pm 0.073$ & $5.789 \pm 0.085$ & $1.11 \pm 0.24$ & $1.37 \pm 0.27$ & 0.152 \\
\hline G023.60+00.00 & 6 & 3.9 & 7.9 & $5.506 \pm 0.048$ & 5.480 & $5.491 \pm 0.099$ & $5.542 \pm 0.114$ & $1.04 \pm 0.26$ & $0.87 \pm 0.23$ & 0.822 \\
\hline
\end{tabular}

Notes. ${ }^{a}$ Number of YSOs.

Table 10. Statistics of the number distributions of the projected separation distance between nearest neighbours in IRDCs.

\begin{tabular}{cccccccc}
\hline \hline Name MSXDC & \multicolumn{2}{c}{ Observed distribution } & \multicolumn{2}{c}{ Random distribution } & & & \\
& {$[\log \rangle_{\text {obs }}$} & $\begin{array}{c}\tilde{r}_{\text {obs }} \\
{[\log \mathrm{AU}]}\end{array}$ & $\begin{array}{c}\langle r\rangle_{\text {ran }} \\
{[\log \mathrm{AU}]}\end{array}$ & $\begin{array}{c}\tilde{r}_{\text {ran }} \\
{[\log \mathrm{AU}]}\end{array}$ & $\langle r\rangle_{\mathrm{obs}} /\langle r\rangle_{\text {ran }}$ & $\tilde{r}_{\text {obs }} / \tilde{r}_{\text {ran }}$ & Prob. \\
\hline G304.74+01.32 & $5.083 \pm 0.058$ & 5.136 & $5.030 \pm 0.111$ & $5.047 \pm 0.110$ & $1.13 \pm 0.33$ & $1.23 \pm 0.31$ & 0.901 \\
G048.65-00.29 & $4.786 \pm 0.046$ & 4.741 & $4.854 \pm 0.108$ & $4.875 \pm 0.108$ & $0.85 \pm 0.23$ & $0.73 \pm 0.18$ & 0.860 \\
G035.39-00.33 & $5.123 \pm 0.093$ & 4.999 & $5.145 \pm 0.125$ & $5.137 \pm 0.161$ & $0.95 \pm 0.34$ & $0.73 \pm 0.27$ & 0.823 \\
G034.43+00.24 & $5.117 \pm 0.036$ & 5.081 & $5.104 \pm 0.128$ & $5.123 \pm 0.143$ & $1.03 \pm 0.31$ & $0.91 \pm 0.30$ & 0.966 \\
G033.69-00.01 & $5.539 \pm 0.019$ & 5.528 & $5.512 \pm 0.100$ & $5.523 \pm 0.119$ & $1.06 \pm 0.25$ & $1.01 \pm 0.28$ & 0.594 \\
G031.97+00.07 & $5.447 \pm 0.066$ & 5.354 & $5.450 \pm 0.128$ & $5.469 \pm 0.148$ & $0.99 \pm 0.33$ & $0.77 \pm 0.26$ & 0.366 \\
G028.53-00.25 & $5.281 \pm 0.077$ & 5.356 & $5.334 \pm 0.118$ & $5.360 \pm 0.123$ & $0.88 \pm 0.29$ & $0.99 \pm 0.28$ & 0.843 \\
G028.37+00.07 & $5.520 \pm 0.073$ & 5.597 & $5.490 \pm 0.096$ & $5.501 \pm 0.113$ & $1.07 \pm 0.30$ & $1.25 \pm 0.32$ & 1.000 \\
G024.33+00.11 & $5.236 \pm 0.040$ & 5.230 & $5.308 \pm 0.117$ & $5.314 \pm 0.148$ & $0.85 \pm 0.24$ & $0.82 \pm 0.28$ & 0.792 \\
G023.60+00.00 & $5.280 \pm 0.057$ & 5.341 & $5.142 \pm 0.148$ & $5.153 \pm 0.172$ & $1.37 \pm 0.50$ & $1.54 \pm 0.61$ & 0.780 \\
\hline
\end{tabular}

the clump masses in the cool northern part $\left(\sim 40-90 M_{\odot}\right)$ are about ten times larger than Jeans masses at $15 \mathrm{~K}$ and the surface density implied by this temperature. Either the Jeans instability has occurred when the cloud has been warmer, i.e. $\sim 30 \mathrm{~K}$, and the clumps have cooled during the contraction, or the dense filament is a result of strong compression by external forces. We note that the filament is thinner at the northeastern end (cross-sectional diameter $\phi \sim 0.45 \mathrm{pc}$ ) than in the southwest $(\phi \sim 1.4 \mathrm{pc})$. Moreover, the filament can have accumulated mass through gravitational inflow from the surrounding cloud (Heitsch et al. 2009).

The present average mass line density in G304.74, $\sim 100 M_{\odot} \mathrm{pc}^{-1}$ exceeds the critical value for a non-magnetic, self-gravitating isothermal cylinder in equilibrium $\left(\sim 25 \mathrm{M}_{\odot} \mathrm{pc}^{-1}\right.$ at $15 \mathrm{~K}$, the critical line density directly proportional to $T_{\text {kin }}$; Ostriker 1964; Curry 2000). For comparison, in several filamentary IRDCs studied by Rathborne et al. (2006; G025.04, G028.53, G028.53, G031.97, G033.69, G034.43, and $\mathrm{G} 035.39)$, the line densities are in the range $\sim 70-800 \mathrm{M}_{\odot} \mathrm{pc}^{-1}$. Supercritical line densities seem to be a common feature in filamentary IRDCs, and they are likely to fragment into smaller cores. Our observations cannot resolve the possible fragments in G304.74, but the process is manisfest in presence of (intermediate- to high-mass) protostars, i.e., the two IRAS sources. On the other hand, the recent interferometric studies of G28.34 (Zhang et al. 2009) and IRDC 19175 (Beuther \& Henning 2009) have provided direct evidence for sub-fragmentation of IRDC clumps. The two studies offer, however, contradictory views of the nature of the underlying instability (turbulent fragmentation vs. Jeans instability).

\subsection{The origin of IRDCs and substructures within them}

IRDCs are the densest parts of molecular cloud complexes. Several observational facts and modelling results suggest that turbulence has an important role in the formation of IRDCs and their fragmentation into clumps. 1) On large scales, molecular clouds have highly supersonic linewidths. In general, these are considered to imply turbulent motions (e.g., Elmegreen \& Scalo 2004; McKee \& Ostriker 2007). However, large linewidths in dense clouds can also be explained by collapsing motions towards local gravitational centres (Heitsch et al. 2009). 2) The filamentary shapes of IRDCs, and molecular clouds in general, are consistent with cloud morphologies predicted by numerical models of supersonic turbulence driven on large scales (e.g., Klessen 2000, 2001; Jappsen et al. 2005). In this model, a dense filament can form where converging flows meet. Also the mass surface density distribution observed in IRDCs have been found to correspond to expectations from numerical simulations of turbulent clouds (Butler \& Tan 2009). 3) The high-mass end of the mass distiribution of a large sample of IRDCs studied by

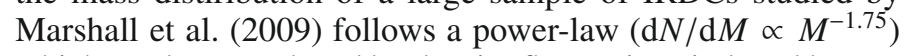
which can be reproduced by density fluctuations induced by turbulence. 4) The mass spectrum of the high-mass clumps within the extensive IRDC sample of RJS06 can be fitted with a powerlaw $\left(\mathrm{d} N / \mathrm{d} M \propto M^{-2.1}\right)$. This power-law agrees with fragmentation due to supersonic turbulence in self-gravitating clouds (Klessen 2001). 5) Finally, the spatial distributions of clumps within IRDCs (cf. Sect. 6.5) show no clear deviation from a random distribution. This is what can be expected if fragmentation is driven by a stochastic process. 
The filamentary stuctures of IRDCs and the fact that star formation in them takes place in clusters suggest that turbulence is driven on large scales (e.g., Klessen 2001). The most likely driving agent for large-scale turbulence is provided by supernova explosions, with an important contribution of density fluctuations caused by older remnants (e.g., Korpi et al. 1998; Joung et al. 2009 and references therein). The Galactic distribution of IRDCs peak in the 5-kpc molecular ring (Simon et al. 2006a; Marshall et al. 2009), where most of the Galactic supernova remnants are found (e.g., Jackson et al. 2008).

The role of turbulence in the fragmention of IRDC clumps into dense cores is less evident. The observed spectral linewidths in clumps are broader than expected from thermal broadening (e.g., Ragan et al. 2006; Sakai et al. 2008; Gibson et al. 2009), but this does not inevitably imply that the gas is turbulent (Heitsch et al. 2009). As discussed in Sect. 6.6, highresolution studies have revealed some regular stuctures within clumps (Zhang et al. 2009; Beuther \& Henning 2009) which point towards gravitational or fluid dynamical instabilities. In any case, self-gravity of shock-compressed clumps or filaments is inherent also in the models of turbulent fragmentation.

\section{Summary and conclusions}

We have mapped IRDC G304.74 in the $870 \mu \mathrm{m}$ dust continuum emission with the APEX telescope. The submm dust continuum observations have been used together with $1.2 \mathrm{~mm}$ data from Beltrán et al. (2006), and archival MSX and IRAS infrared data to derive the physical characteristics of the clumps within the cloud. Besides the dust continuum we used dust extinction data from MSX and 2MASS to derive the $\mathrm{H}_{2}$ column densities, and the mass distribution in the cloud. The results obtained via different methods are in reasonable agreement with each other taking into account the uncertain nature of some of the dust properties and the relation between the $\mathrm{H}_{2}$ column density and extinction. However, the agreement can be improved by assuming an elevated temperature in four clumps near the southwestern end of the cloud, and the possibility of a temperature gradient from about $15 \mathrm{~K}$ in the north and centre to about $20-30 \mathrm{~K}$ in the south cannot be ruled out.

The filamentary cloud G304.74 contains 12 submm clumps. Star formation has already started in the cloud as three of the clumps are associated with both MSX and IRAS point sources. The SEDs of the two IRAS sources indicate bolometric luminosities in the range $\sim 1.5-2 \times 10^{3} L_{\odot}$. These are likely to be intermediate- or high-mass protostars. In addition, one of the clumps (SMM6) is associatied with two MSX $8 \mu \mathrm{m}$ point sources. The remaining eight submm clumps are MIR dark. The masses of these clumps $\left(\sim 40-200 M_{\odot}\right)$ are sufficiently large to enable high-mass star formation, or alternatively, they can represent the cold precursors of proto-clusters. Thus, some of the candidate starless clumps in G304.74 could represent/harbour the so-called high-mass starless cores (HMSCs, e.g., Beuther et al. 2007). Further studies of these high-mass starless clump/core candidates are important in order to constrain the initial conditions of high-mass star and star cluster formation.

The clump masses in G304.74 were compared with the clump mass spectra from more extensive surveys of IRDCs. We found that IRDC clump masses from the present work, and those from Rathborne et al. (2006) and Ragan et al. (2009) probably represent subsamples of the same parent distribution. Also, average distances between a clump and its nearest neighbour in different IRDCs are comparable (within a factor of $\sim 6$ ), suggesting that the fragmentation length-scale does not vary much from cloud to cloud. Moreover, in most IRDCs, clumps seem to be randomly distributed within the cloud area. These characteristics, and the fact the star formation in IRDCs predominantly occurs in the cluster mode, agree with models where fragmentation is driven by large-scale turbulence. It is not clear, yet, how effectual turbulence is for the fragmentation of IRDC clumps into dense cores. High spatial resolution studies, like the ones presented in Zhang et al. (2009) and Beuther \& Henning (2009) have recently started to throw light on scales where gravity is likely to dominate.

Acknowledgements. The authors thank the referee for comments and suggestions which significantly improved the paper. We would like to thank the APEX staff in Chile for performing the LABOCA observations. We acknowledge M. Hennemann for providing the SED fitting tool originally written by J. Steinacker. Furthermore, M. T. Beltrán, P. Bergman, M. J. Butler, J. Kainulainen, H. Linz, and K. Mattila are thanked for useful discussions and suggestions. The authors acknowledge support from the Academy of Finland through grants 117206 and 132291. This research made use of data products from the Midcourse Space Experiment. Processing of the MSX data was funded by the Ballistic Missile Defense Organization with additional support from the NASA Office of Space Science. In addition, this publication makes use of data products from the Two Micron All Sky Survey, which is a joint project of the University of Massachusetts and the Infrared Processing and Analysis Center/California Institute of Technology, funded by the National Aeronautics and Space Administration and the National Science Foundation. This work has made use of the NASA/IPAC Infrared Science Archive, which is operated by the Jet Propulsion Laboratory/California Institute of Technology, under contract with NASA, the NASA Astrophysics Data System, and the VizieR Catalogue access tool (CDS, Strasbourg, France).

\section{References}

Bacmann, A., André, P., Puget, J.-L., et al. 2000, A\&A, 361, 555

Ballesteros-Paredes, J., Klessen, R. S., Mac Low, M.-M., \& Vázquez-Semadeni,

E. 2007, in Protostars and Planets V, ed. B. Reipurth, D. Jewitt, \& K. Keil

(Tucson: Univ. of Arizona Press), 63

Beltrán, M. T., Brand, J., Cesaroni, R., et al. 2006, A\&A, 447, 221

Bergin, E. A., \& Tafalla, M. 2007, ARA\&A, 45, 339

Bertoldi, F., \& McKee, C. F. 1992, ApJ, 395, 140

Bessell, M. S., \& Brett, J. M. 1988, PASP, 100, 1134

Beuther, H., \& Henning, Th. 2009, A\&A, 503, 859

Beuther, H., \& Sridharan, T. K. 2007, ApJ, 668, 348

Beuther, H., \& Steinacker, J. 2007, ApJ, 656, L85

Beuther, H., Schilke, P., Menten, K. M., et al. 2002a, ApJ, 566, 945

Beuther, H., Walsh, A., Schilke, P., et al. 2002b, A\&A, 390, 289

Beuther, H., Sridharan, T. K., \& Saito, M. 2005, ApJ, 634, L185

Beuther, H., Churchwell, E. B., McKee, C. F., \& Tan, J. C. 2007, in Protostars and Planets V, ed. B. Reipurth, D. Jewitt, \& K. Keil (Tucson: Univ. of Arizona Press), 165

Bohlin, R. C., Savage, B. D., \& Drake, J. F. 1978, ApJ, 224, 132

Bronfman, L., Cohen, R. S., Alvarez, H., May, J., \& Thaddeus, P. 1988, ApJ, 324,248

Butler, M. J., \& Tan, J. C. 2009, ApJ, 696, 484

Carey, S. J., Clark, F. O., Egan, M. P., et al. 1998, ApJ, 508, 721

Carey, S. J., Feldman, P. A., Redman, R. O., et al. 2000, ApJ, 543, L157

Cesaroni, R., Walmsley, C. M., Koempe, C., \& Churchwell, E. 1991, A\&A, 252, 278

Chambers, E. T., Jackson, J. M., Rathborne, J. M., \& Simon, R. 2009, ApJS, 181, 360

Chen, X., Launhardt, R., Bourke, T. L., Henning, Th., \& Barnes, P. J. 2008, ApJ, 683,862

Curry, C. L. 2000, ApJ, 541, 831

Dame, T. M., Ungerechts, H., Cohen, R. S., et al. 1987, ApJ, 322, 706

Draine, B. T., Dale, D. A., Bendo, G., et al. 2007, ApJ, 663, 866

Du, F., \& Yang, J. 2008, ApJ, 686, 384

Egan, M. P., Shipman, R. F., Price, S. D., et al. 1998, ApJ, 494, L199

Egan, M. P., Price, S. D., Kraemer, K. E., et al. 2003, VizieR Online Data Catalog, 5114, 0

Ellingsen, S. P. 2006, ApJ, 638, 241

Elmegreen, B. G., \& Scalo, J. 2004, ARA\&A, 42, 211

Evans, N. J., Dunham, M. M., Jørgensen, J. K., et al. 2009, ApJS, 181, 321

Fazal, F. M., Sridharan, T. K., Qiu, K., et al. 2008, ApJ, 688, L41

Fontani, F., Beltrán, M. T., Brand, J., et al. 2005, A\&A, 432, 921

Garay, G., Faúndez, S., Mardones, D., et al. 2004, ApJ, 610, 313 
Gibson, D., Plume, R., Bergin, E., Ragan, S., \& Evans, N. 2009, ApJ, 705, 123 Goldsmith, P. F., \& Langer, W. D. 1978, ApJ, 222, 881

Grave, J. M. C., \& Kumar, M. S. N. 2009, A\&A, 498, 147

Harjunpää, P., \& Mattila, K. 1996, A\&A, 305, 920

Hartmann, L. 2002, ApJ, 578, 914

Heitsch, F., Ballesteros-Paredes, J., \& Hartmann, L. 2009, ApJ, 704, 1735

Hennemann, M., Birkmann, S. M., Krause, O., et al. 2009, ApJ, 693, 1379

Hildebrand, R. H. 1983, QJRAS, 24, 267

Hofner, P., Wyrowski, F., Walmsley, C. M., \& Churchwell, E. 2000, ApJ, 536, 393

Jackson, J. M., Finn, S. C., Rathborne, J. M., et al. 2008, ApJ, 680, 349

Jappsen, A.-K., Klessen, R. S., Larson, R. B., et al. 2005, A\&A, 435, 611

Johnstone, D., Fiege, J. D., Redman, R. O., et al. 2003, ApJ, 588, L37

Joung, M. R., Mac Low, M.-M., \& Bryan, G. L. 2009, ApJ, 704, 137

Kahanpää, J., Mattila, K., Lehtinen, K., Leinert, C., \& Lemke, D. 2003, A\&A, 405, 999

Klessen, R. S. 2000, ApJ, 535, 869

Klessen, R. S. 2001, ApJ, 556, 837

Klessen, R. S., Heitsch, F., \& Mac Low, M.-M. 2000, ApJ, 535, 887

Klessen, R. S., Ballesteros-Paredes, J., Vázquez-Semadeni, E., \& Durán-Rojas, C. 2005, ApJ, 620, 786

Korpi, M. J., Brandenburg, A., \& Tuominen, I. 1998, in Proceedings of the 5th International Workshop "Planetary and Cosmic Dynamos" (Czech Republic: Trest Studia Geoph. et Geod.), 42, 410

Krumholz, M. R., \& McKee, C. F. 2008, Nature, 451, 1082

Lada, C. J., \& Lada, E. A. 2003, ARA\&A, 41, 57

Larson, R. B. 1985, MNRAS, 214, 379

Mac Low, M.-M., \& Klessen, R. S. 2004, Rev. Mod. Phys., 76, 125

Malhotra, S. 1994, ApJ, 433, 687

Marshall, D. J., Robin, A. C., Reylé, C., Schultheis, M., \& Picaud, S. 2006, A\&A, 453, 635

Marshall, D. J., Joncas, G., \& Jones, A. P. 2009, ApJ, 706, 727

Mathis, J. S. 1990, ARA\&A, 28, 37

Matsuyanagi, I., Itoh, Y., Sugitani, K., et al. 2006, PASJ, 58, L29

McKee, C. F., \& Ostriker, E. C. 2007, ARA\&A, 45, 565

McKee, C. F., \& Williams, J. P. 1997, ApJ, 476, 144

Molinari, S., Brand, J., Cesaroni, R., \& Palla, F. 1996, A\&A, 308, 573

Motte, F., Schilke, P., \& Lis, D. C. 2003, ApJ, 582, 277

Muñoz, D. J., Mardones, D., Garay, G., et al. 2007, ApJ, 668, 906

Ormel, C. W., Shipman, R. F., Ossenkopf, V., \& Helmich, F. P. 2005, A\&A, 439, 613
Ossenkopf, V., \& Henning, Th. 1994, A\&A, 291, 943 (OH94)

Ostriker, J. 1964, ApJ, 140, 1056

Padoan, P., Juvela, M., Goodman, A. A., \& Nordlund, ̊̊ 2001, ApJ, 553, 227

Palla, F., Brand, J., Comoretto, G., Felli, M., \& Cesaroni, R. 1991, A\&A, 246, 249

Parsons, H., Thompson, M. A., \& Chrysostomou, A. 2009, MNRAS, 1235

Pérault, M., Omont, A., Simon, G., et al. 1996, A\&A, 315, L165 Peretto, N., \& Fuller, G. A. 2009, A\&A, 505, 405

Pillai, T., Wyrowski, F., Menten, K. M., \& Krügel, E. 2006a, A\&A, 447, 929

Pillai, T., Wyrowski, F., Carey, S. J., et al. 2006b, A\&A, 450, 569

Pillai, T., Wyrowski, F., Hatchell, J., et al. 2007, A\&A, 467, 207

Pineda, J. E., Rosolowsky, E. W., \& Goodman, A. A. 2009, ApJ, 699, L134

Price, S. D., Egan, M. P., Carey, S. J., et al. 2001, AJ, 121, 2819

Ragan, S. E., Bergin, E. A., Plume, R., et al. 2006, ApJS, 166, 567

Ragan, S. E., Bergin, E. A., \& Gutermuth, R. A. 2009, ApJ, 698, 324 (RBG09)

Rathborne, J. M., Jackson, J. M., Chambers, E. T., et al. 2005, ApJ, 630, L181

Rathborne, J. M., Jackson, J. M., \& Simon, R. 2006, ApJ, 641, 389 (RJS06)

Rathborne, J. M., Simon, R., \& Jackson, J. M. 2007, ApJ, 662, 1082

Rathborne, J. M., Jackson, J. M., Zhang, Q., \& Simon, R. 2008, ApJ, 689, 1141

Sakai, T., Sakai, N., Kamegai, K., et al. 2008, ApJ, 678, 1049

Salpeter, E. E. 1955, ApJ, 121, 161

Simon, R., Jackson, J. M., Rathborne, J. M., \& Chambers, E. T. 2006a, ApJ, 639, 227

Simon, R., Rathborne, J. M., Shah, R. Y., et al. 2006b, ApJ, 653, 1325

Siringo, G., Kreysa, E., Kovács, A., et al. 2009, A\&A, 497, 945

Skrutskie, M. F., Cutri, R. M., Stiening, R., et al. 2006, AJ, 131, 1163

Sridharan, T. K., Beuther, H., Schilke, P., et al. 2002, ApJ, 566, 931

Sridharan, T. K., Beuther, H., Saito, M., et al. 2005, ApJ, 634, L57

Stodólkiewicz, J. S. 1963, Acta Astron., 13, 30

Teyssier, D., Hennebelle, P., \& Pérault, M. 2002, A\&A, 382, 624

Thompson, M. A., Hatchell, J., Walsh, A. J., et al. 2006, A\&A, 453, 1003

van der Wiel, M. H. D., \& Shipman, R. F. 2008, A\&A, 490, 655

Vasyunina, T., Linz, H., Henning, Th., et al. 2009, A\&A, 499, 149

Vuong, M. H., Montmerle, T., Grosso, N., et al. 2003, A\&A, 408, 581

Wang, Y., Zhang, Q., Rathborne, J. M., et al. 2006, ApJ, 651, L125

Weingartner, J. C., \& Draine, B. T. 2001, ApJ, 548, 296

Williams, J. P., \& McKee, C. F. 1997, ApJ, 476, 166

Williams, J. P., de Geus, E. J., \& Blitz, L. 1994, ApJ, 428, 693

Wood, D. O. S., \& Churchwell, E. 1989, ApJS, 69, 831

Zhang, Q., Wang, Y., Pillai, T., \& Rathborne, J. 2009, ApJ, 696, 268 